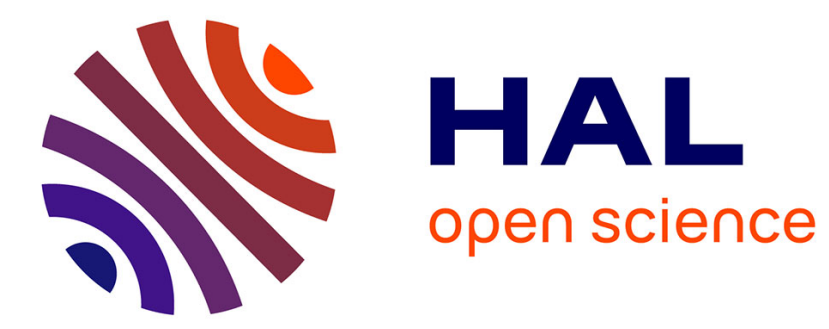

\title{
The image source method for calculating the vibrations of simply supported convex polygonal plates
}

\author{
Jacques Cuenca, François Gautier, Laurent Simon
}

\section{To cite this version:}

Jacques Cuenca, François Gautier, Laurent Simon. The image source method for calculating the vibrations of simply supported convex polygonal plates. Journal of Sound and Vibration, 2009, 322 (4-5), pp.1048-1069. hal-00943860

\section{HAL Id: hal-00943860 https://hal.science/hal-00943860}

Submitted on 9 Feb 2014

HAL is a multi-disciplinary open access archive for the deposit and dissemination of scientific research documents, whether they are published or not. The documents may come from teaching and research institutions in France or abroad, or from public or private research centers.
L'archive ouverte pluridisciplinaire HAL, est destinée au dépôt et à la diffusion de documents scientifiques de niveau recherche, publiés ou non, émanant des établissements d'enseignement et de recherche français ou étrangers, des laboratoires publics ou privés. 


\title{
The image source method for calculating the vibrations of simply supported convex polygonal plates
}

\author{
J. Cuenca, F. Gautier, L. Simon \\ Laboratoire d'Acoustique de l'Université du Maine, UMR CNRS 6613, Avenue Olivier Messiaen, \\ 72085 Le Mans cedex 9, France
}

\begin{abstract}
The aim of this paper is to show that the Image Source Method (ISM) can be used both for analytically calculating Green's functions of particular simply supported convex polygonal plates and for predicting medium and high frequency vibrations of arbitrarily shaped simply supported convex polygonal plates with controllable precision. In the first part of the paper, the method for obtaining the Green's function of a polygonal plate by ISM is developed. Examples for plates of different geometries (rectangle, isosceles right triangle, half-equilateral triangle and equilateral triangle) are given. In the second part of the paper, the pertinence of ISM for predicting medium and high frequency vibrations of arbitrarily shaped simply supported convex polygonal plates is investigated. An approximation based on the exclusion of image sources beyond a certain distance from the receiver is used in order to take advantage of the dissipation of vibrational energy through wave propagation. We investigate the influence of structural damping and truncation distance on the accuracy of such approximation. The computed responses are in good agreement with reference solutions, which are analytically known or obtained by the finite element method.
\end{abstract}

\section{Introduction}

An analysis of literature shows that there is a need of accurate prediction tools in the fields of acoustics and structural dynamics. In medium and high frequency ranges, Finite Element Methods (FEM) often require prohibitive calculation capabilities due to the need of a significant number of elements and to the high modal densities often encountered in structures. Although these methods are continuously extended to higher frequencies by computer hardware improvement, strong limitations still remain. Statistical Energy Analysis (SEA) [1] provides fast estimations of vibrational levels and power flows, for which it certainly remains the most popular method for such purposes. Determining pertinent subsystems, accurately estimating damping ratios and coupling loss factors are the main difficulties for the practical implementation of SEA.

As a consequence, several methods have been proposed, some as extensions of FEM and SEA, and some based on rather different formalisms. Among these, Mace et al. [2] developed an extension of FEM to high frequencies by post-processing finite element models using structure periodicity conditions. Le Bot [3] developed an extension of SEA in which the distribution of energy density inside each sub-system is predicted. Cotoni et al. [4] studied a hybrid FEM-SEA method for the prediction of medium frequency vibrations. Chae et al. [5] constructed a ray tube model with time averaged kinetic and potential energies for predicting vibrational field distributions for simple and coupled plates. They also gave a comprehensive bibliographic review of existent high frequency methods. Ladevèze [6] developed the Variational Theory of Complex Rays, which is based on the expansion of vibrations on a superposition of interior modes, edge modes and corner modes, applicable to medium frequency vibrations. Lastly, references to other improved finite element approaches can be found in [7]. The main inconvenient of methods based on a modal or a finite element description is that the number of modes or elements increases with frequency. 
On the other hand, formulations based on SEA provide quadratic results, so that no accurate prediction of phase phenomena such as interferences is possible.

Otherwise, formulations directly based on wave propagation are adequate for predicting the exact dynamic field in enclosed spaces without being disadvantaged by the large number of modes or elements at high frequencies. Among these, mainly two approaches are relevant: the ray-tracing technique and the Image Source Method (ISM). These methods are widely used in room acoustics $[8,9,10,11,12]$ and consist in predicting the dynamic field of an enclosed domain from the knowledge of the possible propagation paths between the source and an observation point. Such propagation paths can be described either as rays or as virtual sources. The ray-tracing model is often known as an approximation in which the directions of propagation are quantised and the receiver is a spherical volume. On the other hand, ISM consists in replacing the boundaries of the domain by mirror sources of the original source. The weights and locations of image sources are calculated in order to satisfy the boundary conditions of the domain. Moreover, ISM is analytical regardless of the specific shape of boundaries if these form a convex polygon, whereas other analytical methods such as modal analysis [13] or the superposition method [14] are only applicable to simple geometries. In spite of this, ISM has only been applied to academic polygonal geometries in structural dynamics $[15,16,17]$ or used for predicting the vibrations of semi-infinite plates including an heterogeneity $[18,19]$. To the best of our knowledge, the most extensive work on ISM for polygonal plates has been done by Gunda et al. [16], who applied it to rectangular plates with simply supported and roller boundary conditions and observed a rapid convergence towards FEM and exact solutions in medium and high frequencies. However, ISM has not been applied or extended to polygonal plates of other shapes, which is a real need for modelling high frequency vibrations of plates.

The aim of this paper is to test the capability of ISM for predicting medium and high frequency vibrations of simply supported convex polygonal plates with controllable precision. The method allows as well to analytically calculate the Green's functions of plates of particular shape. We restrict our study to simply supported boundary conditions since the associated reflection coefficient is unitary and independent from the angle of incidence of waves. The paper is organised as follows. The general method for obtaining Green's functions from the ISM description is developed and illustrated by several examples in section 2. In section 3, the practical implementation of ISM is presented: The truncation of the image source generation process, which is the main difficulty known for this approach, is analysed and discussed.

\section{Green's functions of convex polygonal plates using the Image Source Method}

The aim of this section is to develop a general method for obtaining Green's functions of polygonal plates from ISM. Statement of the problem and principles of the method are first described.

\subsection{Statement of the problem}

The studied system is a thin plate with an interior domain $\Omega$ and boundaries $\partial \Omega$ defining a convex polygon. The plate is assumed to be excited by an elementary point source at the location $\mathbf{r}_{0}$ (Fig. 1). In the following, harmonic motion is assumed and $e^{-j \omega t}$ time dependence is implicit. Flexural vibrations of the plate are considered in the framework of Kirchhoff's theory. Simply supported edges are assumed, corresponding to zero displacement and zero bending moment boundary conditions on $\partial \Omega$. The Green's function $G_{\Omega}$ associated to the flexural vibrations of the plate is then a solution of the set of equations

$$
\begin{cases}D\left(\nabla^{4}-k_{f}^{4}\right) G_{\Omega}\left(\mathbf{r}, \mathbf{r}_{0}\right)=\delta\left(\mathbf{r}-\mathbf{r}_{0}\right) & \text { in } \Omega \\ G_{\Omega}\left(\mathbf{r}, \mathbf{r}_{0}\right)=0 & \text { on } \partial \Omega \\ \frac{\partial^{2} G_{\Omega}}{\partial n^{2}}\left(\mathbf{r}, \mathbf{r}_{0}\right)+\nu \frac{\partial^{2} G_{\Omega}}{\partial t^{2}}\left(\mathbf{r}, \mathbf{r}_{0}\right)=0 & \text { on } \partial \Omega\end{cases}
$$




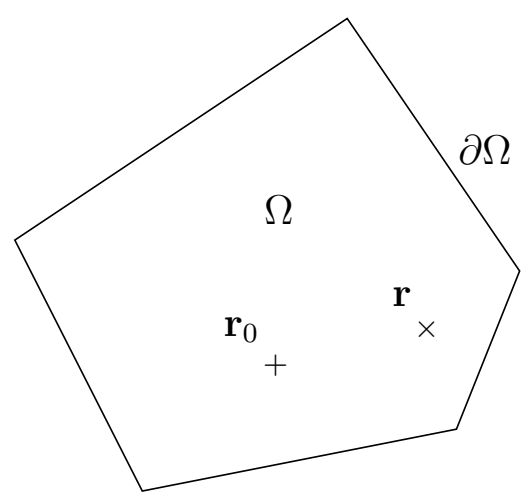

Figure 1: Polygonal plate with inside domain $\Omega$ and boundaries $\partial \Omega$. + , source; $\times$, receiver.

where $D\left(\nabla^{4}-k_{f}^{4}\right)$ is the differential operator governing flexural wave propagation, $\delta$ is the Dirac delta function, $\nu$ is the Poisson's ratio and $n$ and $t$ are respectively the normal and the tangent to the boundary [13]. The flexural wavenumber $k_{f}$ can be written

$$
k_{f}=\left(\omega^{2} \frac{\rho h}{D}\right)^{1 / 4}
$$

where $\rho$ and $h$ are respectively the density and thickness of the plate and

$$
D=\frac{E h^{3}}{12\left(1-\nu^{2}\right)}
$$

is the flexural rigidity of the plate, $E$ being the Young's modulus. Structural losses are assumed to be spread in the medium without any damping mechanism at the boundaries. This can be taken into account by considering the damping ratio $\eta$ as an imaginary part in the Young's modulus, which is written in the form

$$
E=E_{0}(1-\mathrm{j} \eta)
$$

Solving the set of equations (1) is the main target of the following.

\subsection{Modal expansion of the Green's function}

The solution of Eqs. (1) is classically expressed using a normal modal expansion [13], in the form

$$
G_{\Omega}\left(x, y ; x_{0}, y_{0}\right)=\sum_{\mu=1}^{+\infty} \frac{\phi_{\mu}\left(x_{0}, y_{0}\right) \phi_{\mu}(x, y)}{D\left(k_{\mu}^{4}-k_{f}^{4}\right)}
$$

where $\phi_{\mu}$ are real modal shapes, which are normalised to unity and obey the orthogonality relationship

$$
\int_{\Omega} \phi_{\mu} \phi_{\mu^{\prime}}^{*} d \Omega=\delta_{\mu \mu^{\prime}}
$$

The modal shapes are determined as the solutions of the homogeneous set of equations

$$
\begin{cases}D\left(\nabla^{4}-k_{\mu}^{4}\right) \phi_{\mu}(\mathbf{r})=0 & \text { in } \Omega, \\ \phi_{\mu}(\mathbf{r})=0 & \text { on } \partial \Omega, \\ \frac{\partial^{2} \phi_{\mu}}{\partial n^{2}}(\mathbf{r})+\nu \frac{\partial^{2} \phi_{\mu}}{\partial t^{2}}(\mathbf{r})=0 & \text { on } \partial \Omega .\end{cases}
$$


Furthermore, the wavenumber $k_{\mu}$ is linked to the eigenvalue $\omega_{\mu}$ associated to mode $\mu$ by the relationship

$$
\omega_{\mu}=\sqrt{\frac{D_{0}}{\rho h}} k_{\mu}^{2},
$$

where $D_{0}$ is the real part of $D$.

\subsection{Image Source Method (ISM)}

ISM consists in replacing the finite plate $\Omega$, excited by a point source at $\mathbf{r}_{0}$, by an infinite plate containing the original source plus image sources whose locations and amplitude weights are calculated in order to satisfy the boundary conditions $[8,9,12,16]$. The locations of image sources are obtained by performing successive "mirror" reflections of the initial source on the edges $\partial \Omega$ of the plate. Simply supported boundary conditions are considered here, which are a very particular case since the associated reflection coefficient is $\mathcal{R}=-1$, i.e. unitary and independent from the angle of incidence of waves [13]. This implies that the function describing the locations of image sources is a sum of Dirac delta functions, with weights +1 or -1 , according to the parity of reflections. As a convention, such function is here called the image source cloud of the studied system, noted $\mathcal{D}_{\Omega}\left(\mathbf{r}, \mathbf{r}_{0}\right)$ as it is a function of space $\mathbf{r}$ and initial source location $\mathbf{r}_{0}$ and is associated to the shape of the boundaries $\partial \Omega$. An example of the image source cloud of an arbitrarily convex polygonal plate is shown in Fig. 2. The step by step construction of the image sources cloud is given in more detail in Appendix A.

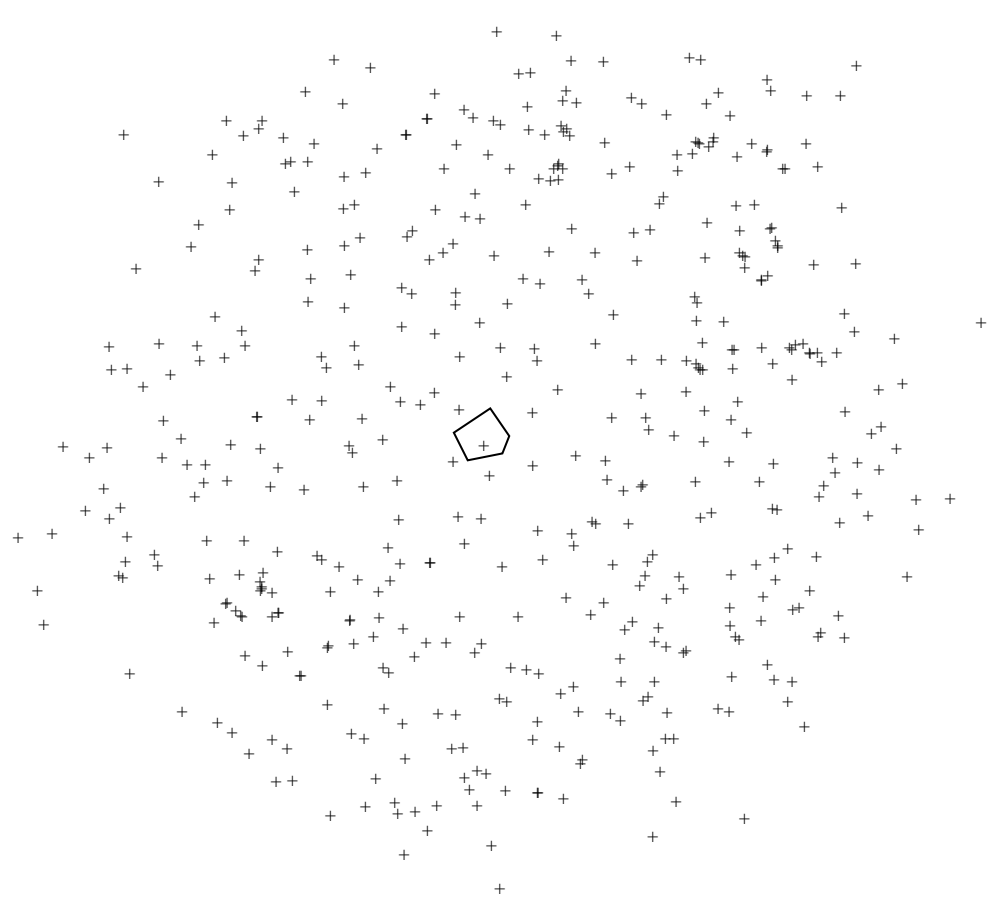

Figure 2: Image source cloud for an arbitrarily convex polygonal plate (first 12 reflections, giving rise to 483 image sources). - - , plate boundaries; + , sources.

Subsequently, the vibrational field at the receiver is obtained as the superposition of elementary contributions from all the sources. Eqs. (1) can then be written in the condensed form

$$
D\left(\nabla^{4}-k_{f}^{4}\right) G_{\Omega}\left(\mathbf{r}, \mathbf{r}_{0}\right)=\mathcal{D}_{\Omega}\left(\mathbf{r}, \mathbf{r}_{0}\right) .
$$

The boundary conditions are satisfied by the existence of an infinity of image sources, represented by the function $\mathcal{D}_{\Omega}\left(\mathbf{r}, \mathbf{r}_{0}\right)$. In order to infer an analytical expression of $G_{\Omega}\left(\mathbf{r}, \mathbf{r}_{0}\right)$, we use the Green's function $G_{\infty}$ 
of the associated infinite plate [16]. For a point source located at $\mathbf{0}, G_{\infty}$ is the solution of the equation

$$
D\left(\nabla^{4}-k_{f}^{4}\right) G_{\infty}(\mathbf{r}, \mathbf{0})=\delta(\mathbf{r})
$$

and can be written as

$$
G_{\infty}(\mathbf{r}, \mathbf{0})=\frac{\mathrm{j}}{8 k_{f}^{2} D}\left(H_{0}^{(1)}\left(k_{f}|\mathbf{r}|\right)-H_{0}^{(1)}\left(\mathrm{j} k_{f}|\mathbf{r}|\right)\right)
$$

where $H_{0}^{(1)}$ is the cylindrical Hankel's function of the first kind of order 0 and $|\mathbf{r}|$ is the source-to-receiver distance [20]. From Eqs. (9) and (10), the Green's function of the convex polygonal plate can be written as

$$
G_{\Omega}\left(\mathbf{r}, \mathbf{r}_{0}\right)=\mathcal{D}_{\Omega}\left(\mathbf{r}, \mathbf{r}_{0}\right) * G_{\infty}(\mathbf{r}, \mathbf{0}) .
$$

In this convolution product, acting on variable $\mathbf{r}$, the infinite space Green's function is initially centered on $\mathbf{0}$, and $\mathcal{D}_{\Omega}$ acts as a translation, weighting and summation operator of the contributions of all sources.

Eq. (12) is the general ISM solution of Eqs. (1) for any simply supported convex polygonal plate. In the following, ISM is applied to plates of particular polygonal shapes that allow to express the Green's function analytically. The practical implementation of ISM and its application to convex polygonal plates of arbitrary shape are discussed in section 3.

\subsection{Obtaining a modal expansion of the Green's function from ISM}

Some special geometries of plates give rise to a spatially periodic image source cloud. The resulting Green's function $G_{\Omega}$ is then spatially periodic itself. Thus, by using Fourier series, $G_{\Omega}$ can be expressed as a function of discrete wavenumbers, which can be interpreted as a modal expansion of the Green's function. The identity between these two kinds of expressions of a function is usually known as the Poisson summation formula (see for example [20]).

The first step for obtaining such expression for the Green's function of the plate is to describe the spatially periodic image source cloud by using two functions, $\mathcal{P}_{\Omega}$ and $\mathcal{E}$, respectively a periodisation operator and an elementary cell. $\mathcal{P}_{\Omega}$ acts as a summation of $\mathcal{E}$ with translations. The image source cloud can then be written as the convolution product

$$
\mathcal{D}_{\Omega}\left(\mathbf{r}, \mathbf{r}_{0}\right)=\mathcal{P}_{\Omega}(\mathbf{r}) * \mathcal{E}\left(\mathbf{r}, \mathbf{r}_{0}\right)
$$

and the periodisation operator can be written in cartesian coordinates as

$$
\mathcal{P}_{\Omega}(x, y)=\sum_{p=-\infty}^{+\infty} \sum_{q=-\infty}^{+\infty} \delta\left(x-p \lambda_{x}, y-q \lambda_{y}\right)
$$

with $\lambda_{x}$ and $\lambda_{y}$ being the spatial periods of the image source cloud. Eq. (12) can then be written as

$$
G_{\Omega}\left(x, y ; x_{0}, y_{0}\right)=\sum_{p=-\infty}^{+\infty} \sum_{q=-\infty}^{+\infty} \delta\left(x-p \lambda_{x}, y-q \lambda_{y}\right) * \mathcal{E}\left(x, y ; x_{0}, y_{0}\right) * G_{\infty}(x, y ; 0,0)
$$

Since $G_{\Omega}$ is periodic, it can be expressed using a Fourier series, in the form

$$
G_{\Omega}\left(x, y ; x_{0}, y_{0}\right)=\sum_{m=-\infty}^{+\infty} \sum_{n=-\infty}^{+\infty} C_{m n} e^{-\mathrm{j}\left(k_{m} x+k_{n} y\right)}
$$

where $k_{m}=m 2 \pi / \lambda_{x}$ and $k_{n}=n 2 \pi / \lambda_{y}$ describe the spatial periodicity of $G_{\Omega}$ and where

$$
C_{m n}=\frac{1}{\lambda_{x} \lambda_{y}} \int_{0}^{\lambda_{x}} \int_{0}^{\lambda_{y}} G_{\Omega}\left(x, y ; x_{0}, y_{0}\right) e^{\mathrm{j}\left(k_{m} x+k_{n} y\right)} d x d y
$$


Eqs. (16) and (17) yield

$$
G_{\Omega}\left(x, y ; x_{0}, y_{0}\right)=\frac{1}{\lambda_{x} \lambda_{y}} \sum_{m=-\infty}^{+\infty} \sum_{n=-\infty}^{+\infty} \mathcal{F}\left[\mathcal{E}\left(x, y ; x_{0}, y_{0}\right)\right]_{\left(k_{m}, k_{n}\right)} \mathcal{F}\left[G_{\infty}(x, y ; 0,0)\right]_{\left(k_{m}, k_{n}\right)} e^{-\mathrm{j}\left(k_{m} x+k_{n} y\right)}
$$

where $\mathcal{F}$ denotes the Fourier transform, and is defined as

$$
\mathcal{F}[f(\mathbf{r})]_{(\mathbf{k})}=\int_{\mathbb{R}^{2}} f(\mathbf{r}) e^{\mathrm{j} \mathbf{k} \cdot \mathbf{r}} d \mathbf{r},
$$

Eq. (18) is consequently an expansion of the Green's function of the plate $G_{\Omega}$ on harmonic functions, i.e. a modal expansion. The equality between Eqs. (15) and (18) shows the equivalence between modal and image source expansions.

\subsection{Examples}

To the best of our knowledge, ISM has only been used for the analytical calculation of Green's functions in the case of beams [15] and one-dimensional acoustic cavities [20], where the image source cloud is a one-dimensional comb. The case of simply supported rectangular plates has been discussed by Gunda et al. [16], who compared ISM computations with a finite number of image sources to the exact solution. Furthermore, the image source cloud of isosceles right triangular, half-equilateral and equilateral triangles have already been qualitatively studied by Cremer and Müller [10]. They have pointed out that the well-organised source pattern results in a number of possible directions of ray propagation in the domain, but no derivation of the Green's functions of such plates has been developed.

One point in common between these plate geometries is that the image source cloud is spatially periodic. In the following examples, ISM is applied to simply supported polygonal plates of various shapes that lead to a periodic image source cloud. The geometries under consideration are: the rectangle, the isosceles right triangle, the half-equilateral triangle and the equilateral triangle. For each case, the elementary cell $\mathcal{E}$ and the periodisation operator $\mathcal{P}_{\Omega}$ are given, leading to analytical expression of the Green's function $G_{\Omega}$, derived from Eq. (18).

\subsubsection{Simply supported rectangular plate}

Fig. 3 shows the image source cloud of a rectangular plate of dimensions $L_{x}$ and $L_{y}$, simply supported at its four edges and excited by a point source at $\left(x_{0}, y_{0}\right)$. The elementary cell can be taken as the subset of four sources located at $\left( \pm x_{0}, \pm y_{0}\right)$. Since the reflexion coefficient is $\mathcal{R}=-1$, the latter is a quadrupole and can be written

$$
\mathcal{E}\left(x, y ; x_{0}, y_{0}\right)=\delta\left(x-x_{0}, y-y_{0}\right)-\delta\left(x-x_{0}, y+y_{0}\right)-\delta\left(x+x_{0}, y-y_{0}\right)+\delta\left(x+x_{0}, y+y_{0}\right) .
$$

The spatial periods of the cloud are $\lambda_{x}=2 L_{x}$ and $\lambda_{y}=2 L_{y}$, so that the periodisation operator, given by Eq. (14), can be written as

$$
\mathcal{P}_{\Omega}(x, y)=\sum_{p=-\infty}^{+\infty} \sum_{q=-\infty}^{+\infty} \delta\left(x-p 2 L_{x}, y-q 2 L_{y}\right)
$$

and the discrete wavenumbers are $k_{m}=m \pi / L_{x}$ and $k_{n}=n \pi / L_{y}$. The Green's function of the rectangular plate obtained by the image source method is then known from Eq. (13). The Fourier transform of $G_{\infty}(x, y ; 0,0)$ can be written as

$$
\mathcal{F}\left[G_{\infty}(x, y ; 0,0)\right]_{\left(k_{m}, k_{n}\right)}=\frac{1}{D\left(\left(k_{m}^{2}+k_{n}^{2}\right)^{2}-k_{f}^{4}\right)} .
$$




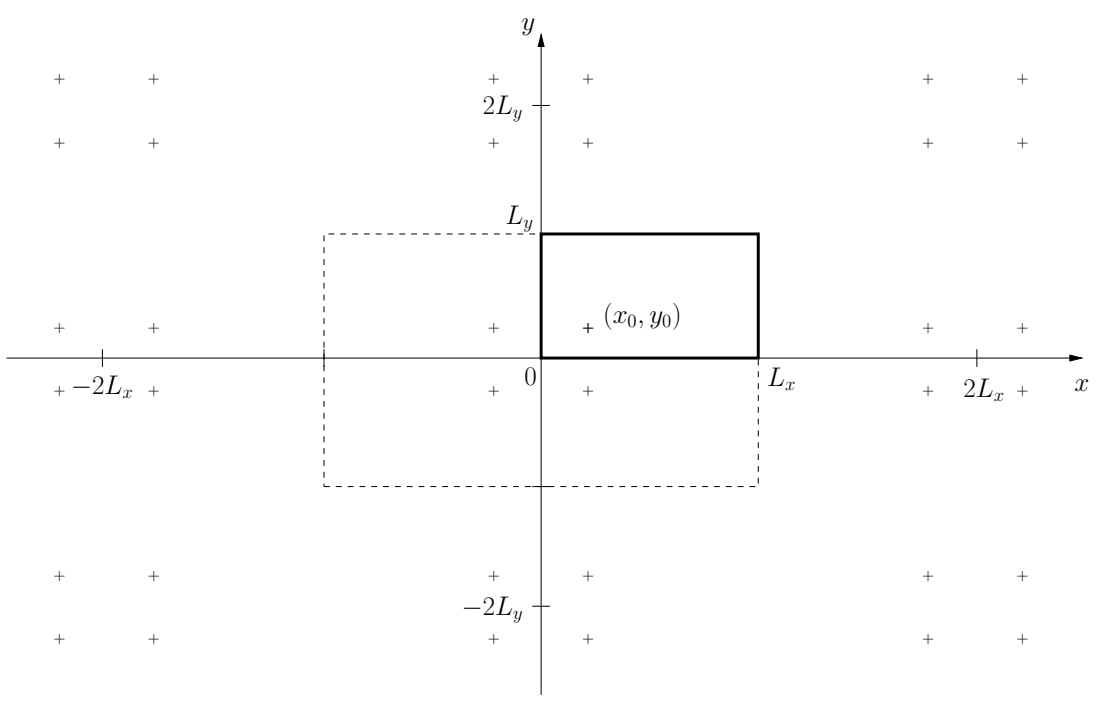

Figure 3: Image source cloud for a rectangular plate. _- plate boundaries; +, sources; ----, elementary cell $\mathcal{E}$.

Furthermore, it can be seen from Eq. (20) that

$$
\mathcal{F}\left[\mathcal{E}\left(x, y ; x_{0}, y_{0}\right)\right]_{\left(k_{m}, k_{n}\right)}=-4 \sin \left(k_{m} x_{0}\right) \sin \left(k_{n} y_{0}\right) .
$$

Inserting (22) and (23) into (18) and rearranging sums on $m$ and $n$ leads to the modal expansion of the Green's function for the rectangular plate,

$$
G_{\Omega}\left(x, y ; x_{0}, y_{0}\right)=\frac{4}{L_{x} L_{y}} \sum_{m=1}^{\infty} \sum_{n=1}^{\infty} \frac{\sin \left(k_{m} x_{0}\right) \sin \left(k_{n} y_{0}\right) \sin \left(k_{m} x\right) \sin \left(k_{n} y\right)}{D\left(\left(k_{m}^{2}+k_{n}^{2}\right)^{2}-k_{f}^{4}\right)} .
$$

Furthermore, the eigenfrequencies of Eq. (8) are given by the zeros of the denominator in Eq. (24)

$$
\omega_{m n}=\left(\frac{D}{\rho h}\right)^{1 / 2}\left(\left(\frac{m \pi}{L_{x}}\right)^{2}+\left(\frac{n \pi}{L_{y}}\right)^{2}\right),
$$

for $(m \geq 1, n \geq 1)$. Eq. (24) is the well-known solution for the rectangular plate [13, 14]. This simple example shows that ISM allows to obtain the Green's function for this academic geometry by using a rather different approach.

\subsubsection{Simply supported isosceles right triangular plate}

The image source cloud of the isosceles right triangular plate of side $L$ with a point source at $\left(x_{0}, y_{0}\right)$ is represented in Fig. 4. The elementary cell can be separated into two quadrupoles of sources respectively located at $\left( \pm x_{0}, \pm y_{0}\right)$ and $\left( \pm\left(L-y_{0}\right), \pm\left(L-x_{0}\right)\right)$. The response of this plate can thus be regarded as the superposition of responses of a square plate to opposite excitations at $\left(x_{0}, y_{0}\right)$ and $\left(L-y_{0}, L-x_{0}\right)$, which was used by Szilard [21] for calculating the static deflection of this plate.

The elementary cell $\mathcal{E}$ and its Fourier transform can be respectively written as

$$
\mathcal{E}\left(x, y, x_{0}, y_{0}\right)=\left\{\begin{array}{l}
\delta\left(x-x_{0}, y-y_{0}\right) \\
-\delta\left(x-x_{0}, y+y_{0}\right) \\
-\delta\left(x+x_{0}, y-y_{0}\right) \\
+\delta\left(x+x_{0}, y+y_{0}\right)
\end{array} \quad+\left\{\begin{array}{l}
-\delta\left(x-\left(L-y_{0}\right), y-\left(L-x_{0}\right)\right) \\
+\delta\left(x-\left(L-y_{0}\right), y+\left(L-x_{0}\right)\right) \\
+\delta\left(x+\left(L-y_{0}\right), y-\left(L-x_{0}\right)\right) \\
-\delta\left(x+\left(L-y_{0}\right), y+\left(L-x_{0}\right)\right)
\end{array}\right.\right.
$$




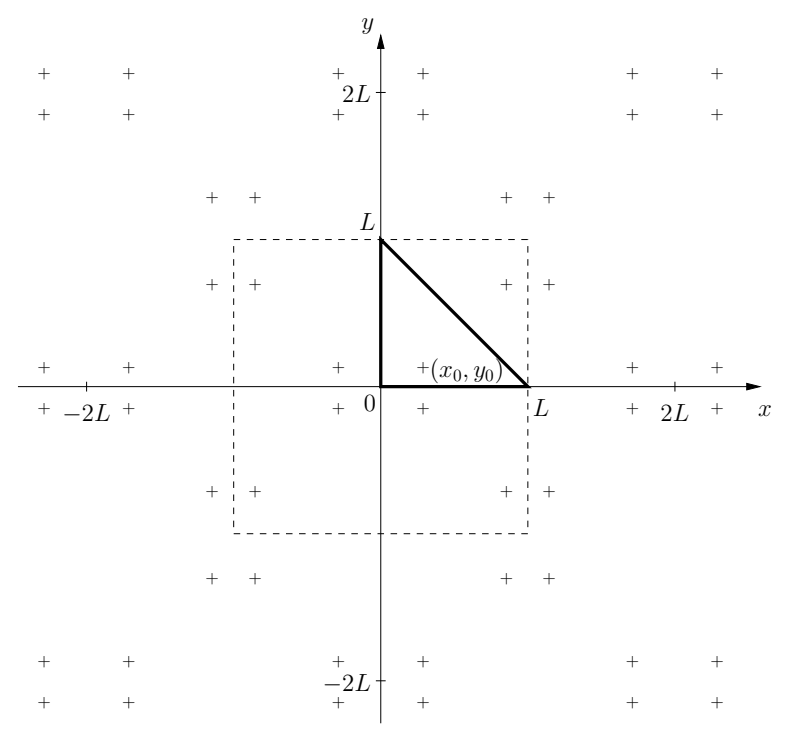

Figure 4: Image source cloud for the isosceles right triangular plate. - , plate boundaries; +, sources; ----, elementary cell $\mathcal{E}$.

and

$$
\mathcal{F}\left[\mathcal{E}\left(x, y, x_{0}, y_{0}\right)\right]_{\left(k_{m}, k_{n}\right)}=-4 \sin \left(k_{m} x_{0}\right) \sin \left(k_{n} y_{0}\right)+4 \sin \left(k_{m}\left(L-y_{0}\right)\right) \sin \left(k_{n}\left(L-x_{0}\right)\right)
$$

The spatial periods of the cloud are $\lambda_{x}=2 L$ and $\lambda_{y}=2 L$, leading to the discrete wavenumbers $k_{m}=$ $m \pi / L$ and $k_{n}=n \pi / L$ and to the periodisation operator

$$
\mathcal{P}_{\Omega}(x, y)=\sum_{p=-\infty}^{+\infty} \sum_{q=-\infty}^{+\infty} \delta(x-p 2 L, y-q 2 L) .
$$

The Green's function $G_{\infty}$ of the infinite plate is the same as the one used in section 2.5.1. By, inserting Eqs. (22) and (27) into Eq. (18), the Green's function for the simply supported isosceles right triangular plate can be expressed as

$$
\begin{aligned}
& G_{\Omega}\left(x, y, x_{0}, y_{0}\right)= \\
& \quad \frac{4}{L^{2}} \sum_{m=1}^{+\infty} \sum_{n=1}^{+\infty} \frac{\sin \left(k_{m} x_{0}\right) \sin \left(k_{n} y_{0}\right)-\sin \left(k_{m}\left(L-y_{0}\right)\right) \sin \left(k_{n}\left(L-x_{0}\right)\right)}{D\left(\left(k_{m}^{2}+k_{n}^{2}\right)^{2}-k_{f}^{4}\right)} \sin \left(k_{m} x\right) \sin \left(k_{n} y\right) .
\end{aligned}
$$

The zeros of the denominator in Eq. (29) are

$$
\omega_{m n}=\left(\frac{D}{\rho h}\right)^{1 / 2}\left(\left(\frac{m \pi}{L}\right)^{2}+\left(\frac{n \pi}{L}\right)^{2}\right),
$$

for $(m \geq 1, n \geq 1)$, which are the same as those in Eq. (25) for $L_{x}=L_{y}=L$. However, the numerator in Eq. (29) consists in a sum of two terms and therefore is cancelled for $m=n$, so that $\omega_{m m}$ are not singularities of $G_{\Omega}$ (i.e. eigenfrequencies).

\subsubsection{Simply supported half-equilateral triangular plate}

The image source cloud of the simply supported half-equilateral triangular plate of vertices $(0,0),(L, 0)$ and $(0, L / \sqrt{3})$ with a point source at $\left(x_{0}, y_{0}\right)$ is represented in Fig. 5. 


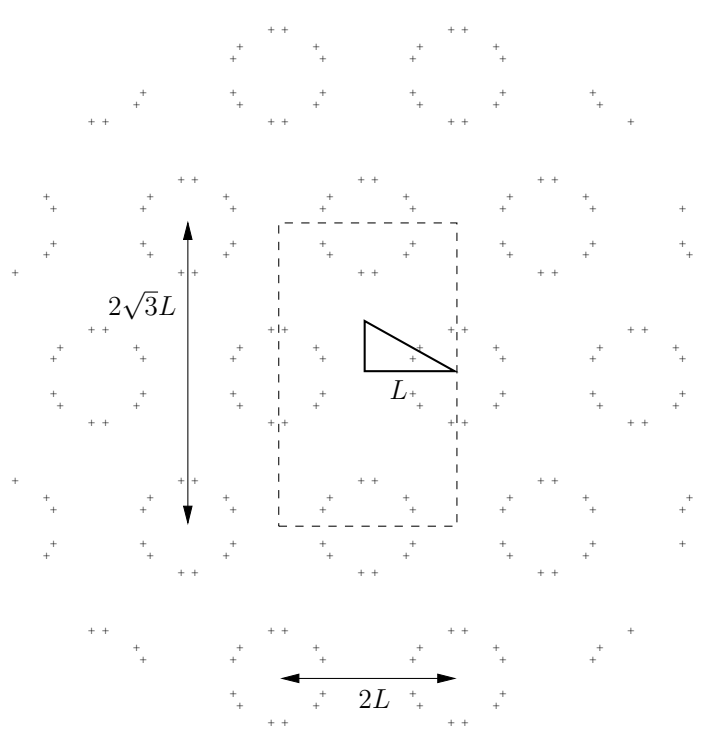

Figure 5: Image source cloud for the half-equilateral triangular plate. ——, plate boundaries; +, sources; ----, elementary cell $\mathcal{E}$.

The elementary cell $\mathcal{E}$ of the image source cloud can be written as the superposition of six quadrupoles of sources:

$$
\mathcal{E}\left(x, y, x_{0}, y_{0}\right)=\sum_{i=1}^{6} \xi_{i}\left\{\begin{array}{l}
\delta\left(x-x_{i}, y-y_{i}\right) \\
-\delta\left(x-x_{i}, y+y_{i}\right) \\
-\delta\left(x+x_{i}, y-y_{i}\right) \\
+\delta\left(x+x_{i}, y+y_{i}\right)
\end{array}\right.
$$

where $\xi_{i}= \pm 1$ is a sequence giving the relative weight of these quadrupoles and $\left( \pm x_{i}, \pm y_{i}\right)(i=1, \ldots, 6)$ are the locations of the sources of each quadrupole (see Appendix B.1 for the detailed expressions of $\left(x_{i}, y_{i}\right)$ and $\left.\xi_{i}\right)$. Fourier Transform of $\mathcal{E}$ gives

$$
\mathcal{F}\left[\mathcal{E}\left(x, y, x_{0}, y_{0}\right)\right]_{\left(k_{m}, k_{n}\right)}=-4 \sum_{i=1}^{6} \xi_{i} \sin \left(k_{m} x_{i}\right) \sin \left(k_{n} y_{i}\right)
$$

Furthermore, the image source cloud is $2 L$-periodic along $x$ and $2 \sqrt{3} L$-periodic along $y$, leading to $k_{m}=$ $m \pi / L$ and $k_{n}=n \pi / \sqrt{3} L$ and

$$
\mathcal{P}_{\Omega}(x, y)=\sum_{p=-\infty}^{+\infty} \sum_{q=-\infty}^{+\infty} \delta(x-p 2 L, y-q 2 \sqrt{3} L)
$$

Application of Eq. (18) consequently leads to the Green's function for the simply supported halfequilateral plate

$$
G_{\Omega}\left(x, y, x_{0}, y_{0}\right)=\frac{4}{\sqrt{3} L^{2}} \sum_{m=1}^{+\infty} \sum_{n=1}^{+\infty} \frac{\sum_{i=1}^{6}-\xi_{i} \sin \left(k_{m} x_{i}\right) \sin \left(k_{n} y_{i}\right)}{D\left(\left(k_{m}^{2}+k_{n}^{2}\right)^{2}-k_{f}^{4}\right)} \sin \left(k_{m} x\right) \sin \left(k_{n} y\right) .
$$

The zeros of the denominator in Eq. (34) are

$$
\omega_{m n}=\left(\frac{D}{\rho h}\right)^{1 / 2}\left(\left(\frac{m \pi}{L}\right)^{2}+\left(\frac{n \pi}{\sqrt{3} L}\right)^{2}\right)
$$


for $(m \geq 1, n \geq 1)$, and are the same as those in Eq. (25) for $L_{x}=L$ and $L_{y}=\sqrt{3} L$. As for the case of the isosceles right triangular plate, the circular frequencies $\omega_{m n}$ for which the numerator in Eq. (34) is cancelled are not eigenfrequencies.

\subsubsection{Simply supported equilateral triangular plate}

The image source cloud of the simply supported equilateral triangular plate of vertices $(0,0),(L, 0)$ and $(L / 2, \sqrt{3} L / 2)$ is represented in Fig. 6 . The elementary cell of the cloud can be written as

$$
\mathcal{E}\left(x, y, x_{0}, y_{0}\right)=\sum_{i=1}^{12} \xi_{i} \delta\left(x-x_{i}, y-y_{i}\right)
$$

where $\left(x_{i}, y_{i}\right)(i=1, \ldots, 12)$ are the locations of the 12 sources that form the elementary cell and $\xi_{i}= \pm 1$ is a sequence giving the weight of each one of these sources (see Appendix B.2 for the detailed expressions of $\left(x_{i}, y_{i}\right)$ and $\left.\xi_{i}\right)$.

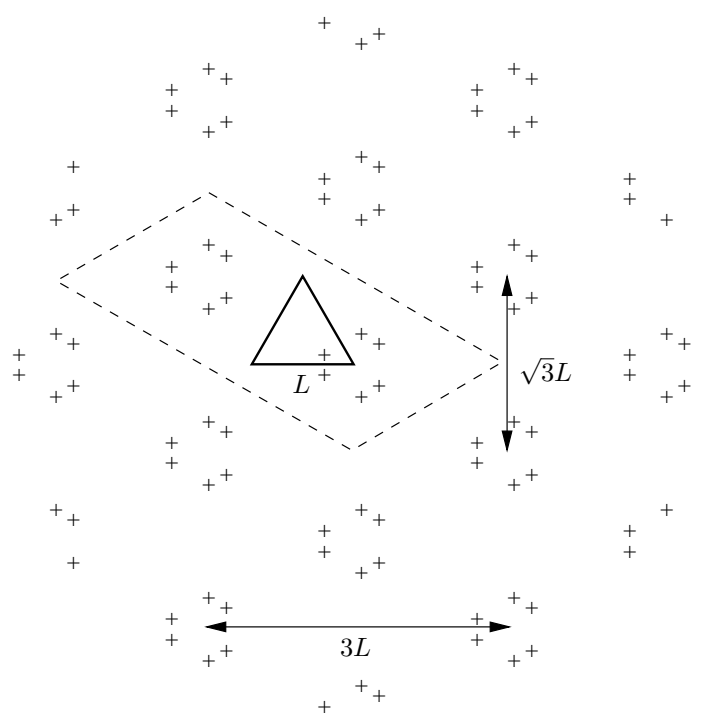

Figure 6: Image source cloud for the equilateral triangular plate. - , plate boundaries; +, sources; ----, elementary cell $\mathcal{E}$.

The spatial periods of the cloud are $\lambda_{x}=3 L$ and $\lambda_{y}=\sqrt{3} L$, leading to $k_{m}=m 2 \pi / 3 L$ and $k_{n}=$ $n 2 \pi / \sqrt{3} L$ and

$$
\mathcal{P}_{\Omega}(x, y)=\sum_{p=-\infty}^{+\infty} \sum_{q=-\infty}^{+\infty} \delta(x-p 3 L, y-q \sqrt{3} L) .
$$

According to Eq. (18), the Green's function of the plate is then

$$
G_{\Omega}\left(x, y, x_{0}, y_{0}\right)=\frac{1}{3 L \cdot \sqrt{3} L} \sum_{m=-\infty}^{+\infty} \sum_{n=-\infty}^{+\infty} \frac{\sum_{i=1}^{12} \xi_{i} e^{\mathrm{j} k_{m} x_{i}} e^{\mathrm{j} k_{n} y_{i}}}{D\left(\left(k_{m}^{2}+k_{n}^{2}\right)^{2}-k_{f}^{4}\right)} e^{-\mathrm{j} k_{m} x} e^{-\mathrm{j} k_{n} y}
$$


which can be written in the form

$$
\begin{aligned}
G_{\Omega}\left(x, y, x_{0}, y_{0}\right)= & \frac{4}{3^{3 / 2} L^{2}} \sum_{m=1}^{+\infty} \sum_{n=1}^{+\infty} \frac{\sum_{i=1}^{12} \xi_{i} \cos \left(k_{m}\left(x_{i}-x\right)\right) \cos \left(k_{n}\left(y_{i}-y\right)\right)}{D\left(\left(k_{m}^{2}+k_{n}^{2}\right)^{2}-k_{f}^{4}\right)} \\
& +\frac{2}{3^{3 / 2} L^{2}} \sum_{m=1}^{+\infty} \frac{\sum_{i=1}^{12} \xi_{i} \cos \left(k_{m}\left(x_{i}-x\right)\right)}{D\left(k_{m}^{4}-k_{f}^{4}\right)} \\
& +\frac{2}{3^{3 / 2} L^{2}} \sum_{n=1}^{+\infty} \frac{\sum_{i=1}^{12} \xi_{i} \cos \left(k_{n}\left(y_{i}-y\right)\right)}{D\left(k_{n}^{4}-k_{f}^{4}\right)} .
\end{aligned}
$$

The zeros of the denominator in Eq. (38) are given by

$$
\omega_{m n}=\left(\frac{D}{\rho h}\right)^{1 / 2}\left(\left(\frac{m 2 \pi}{3 L}\right)^{2}+\left(\frac{n 2 \pi}{\sqrt{3} L}\right)^{2}\right)
$$

for $(m \geq 0, n \geq 0)$ except $(m=0, n=0)$. Here again, the circular frequencies for which the numerator in Eq. (38) is cancelled are not eigenfrequencies.

From this section, we can draw the following conclusions. The Green's function of a polygonal plate can be computed by ISM. In the case of a periodic image source cloud $\mathcal{D}_{\Omega}$, an analytical expansion of the Green's function of the plate $G_{\Omega}$ can be obtained by means of a Poisson summation formula. It should be noticed that this analytical expansion can be obtained for all polygonal shapes which lead to periodic image source cloud.

\section{Effect of truncation of the image source cloud}

The aim of this section is to quantify the accuracy of the plate response calculated from a truncated image source cloud. On one hand, the main drawback for practical implementation of ISM is the need of an infinite number of image sources. On the other hand, a wave propagating in a real-world medium is subjected to geometrical attenuation and structural damping. As a consequence, image sources located far from the receiver do not significantly contribute to the vibrational field. An approximated formulation may then be considered by eliminating all image sources located outside an arbitrary radius from the receiver, as usually defined in room acoustics as the speed of sound times the reverberation time [8]. The flexural vibrations of polygonal plates can then be accurately described with a finite number of image sources and the precision of the estimation can be controlled. We henceforth refer to this formulation as the Approximate Image Source Method (AISM).

\subsection{Truncation of the image source cloud}

In order to investigate the influence of frequency, damping and source-to-receiver distance on wave propagation, the Green's function of an infinite plate, given in Eq. (11), can be asymptotically approximated following [20]

$$
G_{\infty}\left(\mathbf{r}, \mathbf{r}_{s}\right) \simeq \tilde{G}_{\infty}\left(\mathbf{r}, \mathbf{r}_{s}\right)=-\frac{1}{8 \mathrm{j} k_{f}^{2} D}\left(\frac{2}{\pi k_{f}\left|\mathbf{r}-\mathbf{r}_{s}\right|}\right)^{1 / 2} e^{\mathrm{j}\left(k_{f}\left|\mathbf{r}-\mathbf{r}_{s}\right|-\frac{\pi}{4}\right)},
$$

for $\operatorname{Re}\left(k_{f}\right)\left|\mathbf{r}-\mathbf{r}_{s}\right| \gg 1$, where $\mathbf{r}_{s}$ and $\mathbf{r}$ are respectively the locations of a source and a receiver in an infinite plate. In this formulation, evanescent waves are ignored. The modulus of $\tilde{G}_{\infty}$ decreases with increasing circular frequency $\omega$, source-to-receiver distance $\left|\mathbf{r}-\mathbf{r}_{s}\right|$, and structural damping ratio $\eta$, according to

$$
\left|\tilde{G}_{\infty}\left(\mathbf{r}, \mathbf{r}_{s}\right)\right| \sim \omega^{-5 / 4}\left|\mathbf{r}-\mathbf{r}_{s}\right|^{-1 / 2}\left(1+\eta^{2}\right)^{-3 / 16}
$$




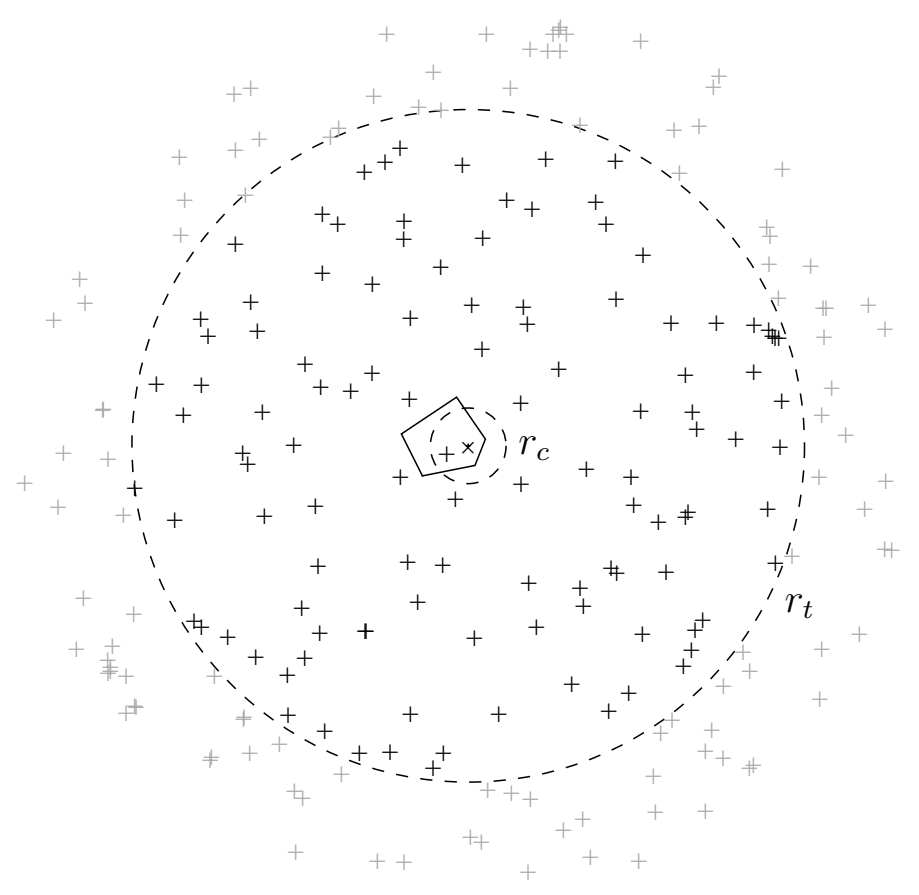

Figure 7: Example of image source cloud truncation for AISM. —-, plate boundaries; ----, characteristic and truncation circles of radii $r_{c}$ and $r_{t}$ centered on the receiver; + , sources taken into account for AISM; + , ignored image sources.

The truncation radius is defined as the distance $r_{t}=\left|\mathbf{r}-\mathbf{r}_{s}\right|$ over which the amplitude of a flexural wave becomes $P$ times lower than for a propagation distance of reference $r_{c}$ (Fig. 7).

This criterion can be expressed as

$$
\left|G_{\infty}\left(0, r_{t}\right)\right|=\frac{\left|G_{\infty}\left(0, r_{c}\right)\right|}{P} .
$$

The choice of the distance $r_{c}$ is arbitrary and may be taken as a characteristic length of the plate. We use the mean free path [1], which is the average distance between two successive image sources, defined as

$$
r_{c}=\frac{\pi S}{p}
$$

where $S$ and $p$ are respectively the total area and the perimeter of the plate. The distance $r_{t}$ obtained from Eq. (43) is used for stopping the image source generation process. In order to control the truncation radius regardless of the geometry of the plate, we use the dimensionless parameter

$$
\gamma=\frac{r_{t}}{r_{c}}
$$

Using the asymptotic expression (41) for $\left|G_{\infty}\left(0, r_{t}\right)\right|$ and for $\left|G_{\infty}\left(0, r_{c}\right)\right|$ in Eq. (43) allows to write

$$
P \simeq \sqrt{\gamma}
$$

Thus, equations (43) and (45) are equivalent definitions of the truncation radius from arbitrary values of $\gamma$ or $P$, such that $\gamma=+\infty$ and $P=+\infty$ correspond to the ISM solution.

\subsection{Influence of structural damping on the accuracy of AISM}

Since the vibrational energy of plates is dissipated during wave propagation, for a given truncation radius $r_{t}$, the accuracy of AISM increases with structural damping. We use the concept of Modal Overlap Factor 
(MOF) as an indicator of the effects of damping on the behaviour of the physical system. The MOF is commonly used in room acoustics and structural dynamics for determining the threshold between low and high frequency ranges [22]. It estimates the number of modes that overlap at a given frequency as the ratio between the $-3 \mathrm{~dB}$ bandwidth $\Delta \omega_{\mu}$ of a resonance and the frequency interval $\delta \omega_{\mu}$ between two successive resonances, as

$$
\mathrm{MOF}=\frac{\Delta \omega_{\mu}}{\delta \omega_{\mu}} .
$$

For vibrating systems, the MOF generally increases with damping and frequency. The value for determining the threshold between low and high frequency ranges results from a semi-empirical observation of the behaviour of the system under consideration [22]. The value MOF $=1$ corresponds to a frequency for which the spacing between eigenfrequencies is equal to the $-3 \mathrm{~dB}$ bandwidth of resonances. This is consequently the frequency for which modes begin to overlap, here taken as the threshold between low and mid frequency ranges. Moreover, the value $\mathrm{MOF}=3$ is chosen as the threshold between mid and high frequency ranges, such that an average of three successive resonances is in a resonance bandwidth. In room acoustics, the frequency for which $\mathrm{MOF}=3$ is refered to as the Schroeder frequency [23].

In order to study the accuracy of AISM in low, mid and high frequency ranges, simulations are performed on a simply supported isosceles right triangular $2 \mathrm{~mm}$-thick steel plate, with Young's modulus $E_{0}=210 \mathrm{GPa}$, Poisson's ratio $\nu=0.3$, density $\rho=7850 \mathrm{~kg} \cdot \mathrm{m}^{-3}$ and for two different structural damping ratios, $\eta=0.03$ and $\eta=0.05$. The vertices are located at $(0,0),(L, 0)$ and $(0, L)$, where $L=1 \mathrm{~m}$. The source and the receiver are respectively located at $\mathbf{r}_{0}=(0.2 L, 0.1 L)$ and $\mathbf{r}=(0.6 L, 0.3 L)$. Fig. 8 shows the corresponding image source cloud, truncated for $\gamma=10.76$.

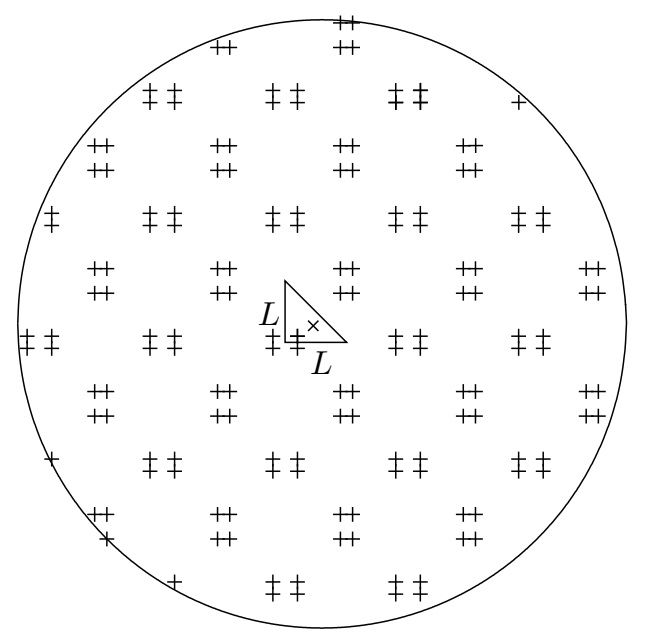

Figure 8: Image source cloud of the isosceles right triangular plate, for the truncation parameter $\gamma=10.76$ $\left(r_{t}=4.95 \mathrm{~m}\right)$, containing 154 sources.

The MOF is usually calculated from the average spacing between resonances over the whole frequency range [1]. Nevertheless, for thin plates, eigenfrequencies are not equally spaced, and then the MOF does not follow a monotonic law of frequency. In particular, symmetries in the geometry induce multiple modes, also called degenerate modes, which lead to an infinite MOF at the corresponding eigenfrequency. Therefore, in order to estimate the low-mid and mid-high frequency thresholds, a smoothed curve is used by considering the local minimum of the MOF over a sliding window of 10 modes and then by fitting it to a first order polynomial in a least-squares sense. This underestimates the value of the modal overlap factor and consequently overestimates the values of the low-medium and medium-high frequency thresholds. Fig. 9 shows the MOF and its smoothed estimation as a function of frequency for $\eta=0.03$ and $\eta=0.05$, with the threshold frequencies corresponding to $\mathrm{MOF}=1$ and $\mathrm{MOF}=3$. 


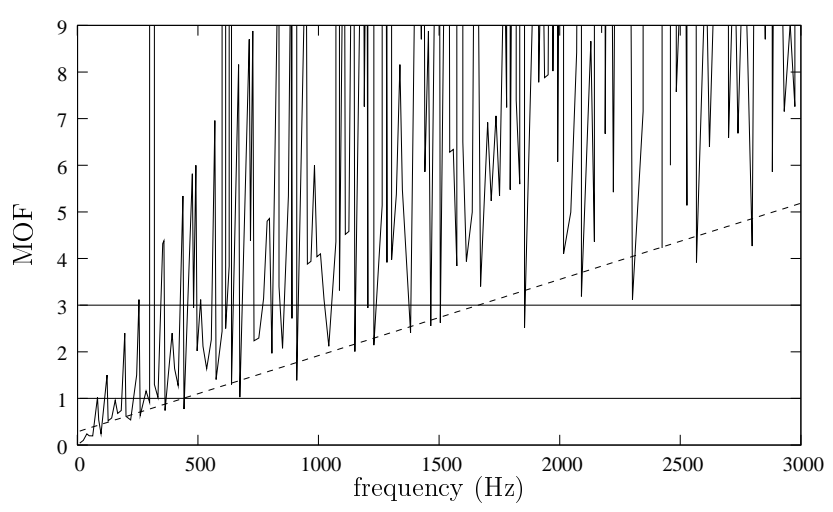

(a)

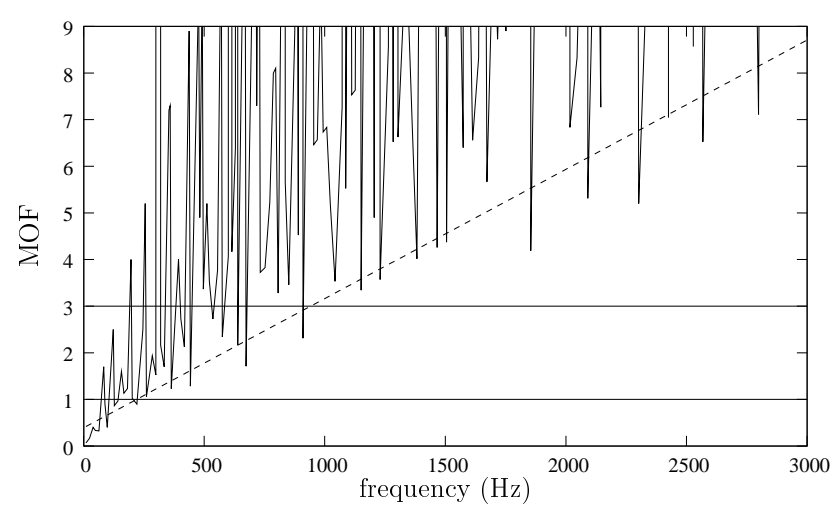

(b)

Figure 9: Modal Overlap Factor of the isosceles right triangular plate for two values of the structural damping ratio: (a) $\eta=3 \cdot 10^{-2}$; (b) $\eta=5 \cdot 10^{-2}$. -

In order to evaluate the differences between AISM and the exact solution, the error at a particular frequency is estimated as

$$
\text { error }=\left|\frac{G_{\Omega}^{(\mathrm{AISM})}-G_{\Omega}^{(\mathrm{ref})}}{G_{\Omega}^{(\mathrm{ref})}}\right|,
$$

where $G_{\Omega}^{(\text {ref })}$ is the exact solution of Eq. (29), calculated by modal expansion. Fig. 10 shows the modulus and the phase of AISM and exact solutions, and the associated error as a function of frequency. The estimated MOF thresholds delimit the three frequency ranges of the response, for which three different degrees of accuracy are obtained. The comparison between Figs. 10(a,c,e) and Figs. 10(b,d,f) shows that the accuracy of AISM is improved for medium and high frequency ranges as structural damping is increased. In particular, good agreeement between AISM and the exact solution is reached in the modulus and in the phase where the response curve presents smooth variations. On the other hand, the accurate reconstruction of resonances and anti-resonances is especially difficult since it needs the interferences between a large number of image sources. 


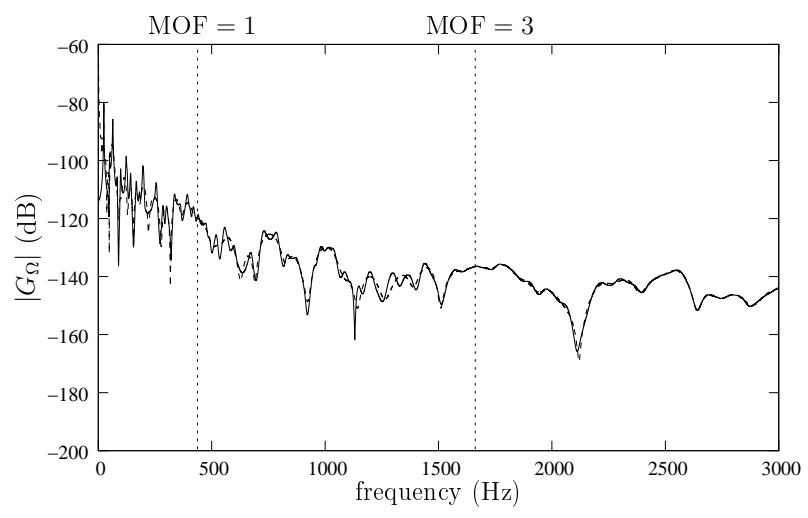

(a)

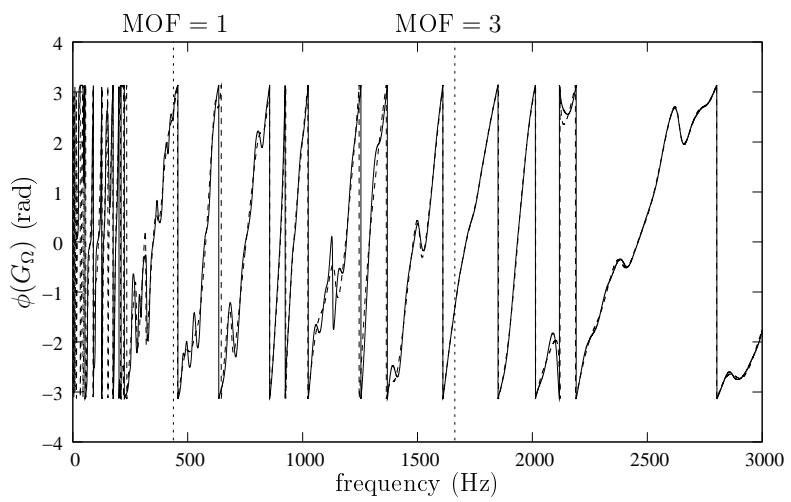

(c)

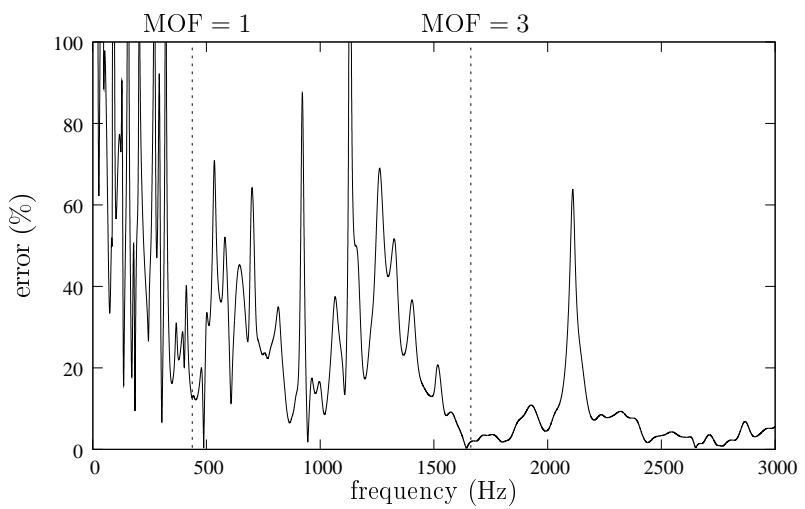

(e)

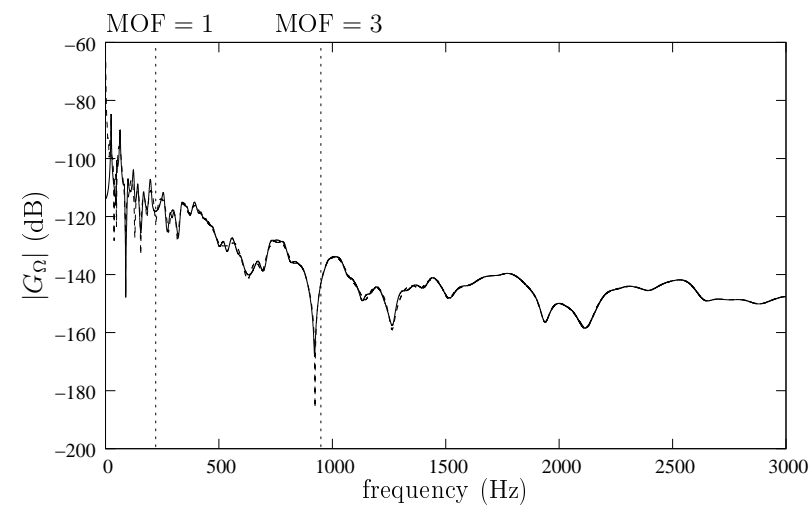

(b)

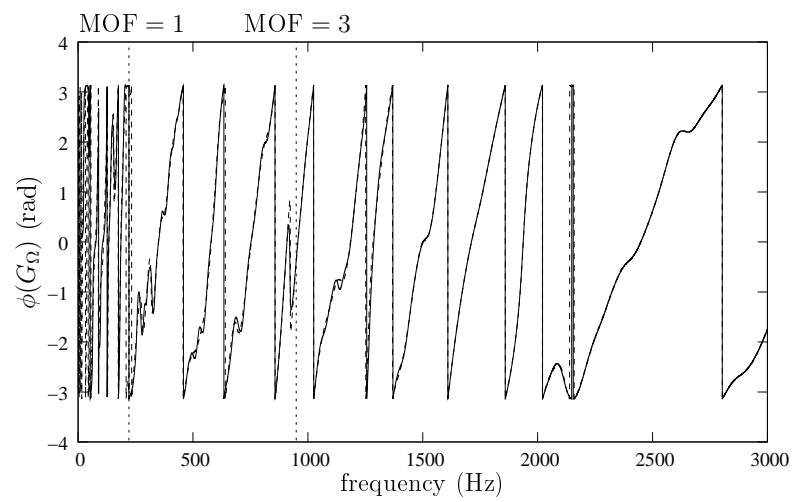

(d)

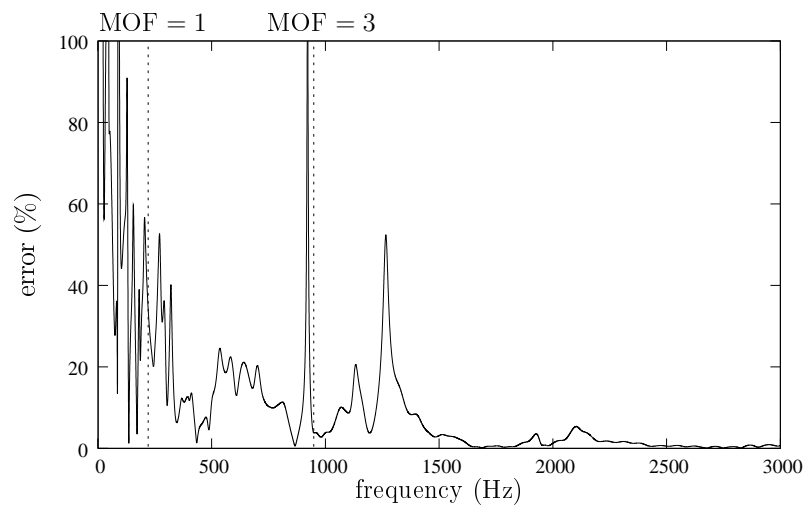

(f)

Figure 10: Frequency response function of the simply supported isosceles right triangular plate between source $\mathbf{r}_{0}$ and receiver $\mathbf{r}$, calculated by modal expansion and by AISM for the truncation parameter $\gamma=10.76$, for two values of the structural damping ratio: (a) modulus, (c) phase and (e) error for $\eta=3 \cdot 10^{-2}$; (b) modulus, (d) phase and (f) error for $\eta=5 \cdot 10^{-2} \cdot-$, exact solution; - - - , AISM. 
The accuracy of modal methods is known to behave oppositely with respect to damping. From a frequency point of view, damping widens resonances, which compels the consideration of a greater number of terms when implementing modal methods. From a spatial point of view, increasing damping reduces the contribution from sources located far from the receiver, and thus improves the accuracy of AISM. Furthermore, as the effect of damping is stronger at high frequencies than at low frequencies, the accuracy of AISM is improved with frequency.

\subsection{Application of AISM to an arbitrary polygonal plate}

In this section, a plate without any symmetry or particular geometrical property is studied. Simulations from AISM and Finite Element Method (FEM) are compared. The plate we use has the same material properties as those in 3.2, with a structural damping ratio $\eta=0.07$. The vertices of the plate are located at $(0,0),(0.75,0)$ and $(0.5625,0.9)$ (in meters). FEM tests are performed using I-DEAS 12 NX (R), with linear interpolated quadrilateral elements. The average element length of the mesh is $3 \mathrm{~mm}$, 18.6 times smaller than the wavelength of the highest eigenfrequency taken into account, i.e. $6332.9 \mathrm{~Hz}$ (mode number 320). The model has 40078 elements and 40534 nodes. For the AISM simulation, the characteristic length is $r_{c}=0.39 \mathrm{~m}$. The truncation parameter is taken as $\gamma=22.44$, which gives a truncation radius of $r_{t}=8.71 \mathrm{~m}$, yielding 793 sources. Fig. 11 shows the plate with the locations of the sources and the receiver. For the damping ratio $\eta=0.07$, the $\mathrm{MOF}=1$ and $\mathrm{MOF}=3$ frequency thresholds are respectively obtained at $248.2 \mathrm{~Hz}$ and $682.6 \mathrm{~Hz}$.

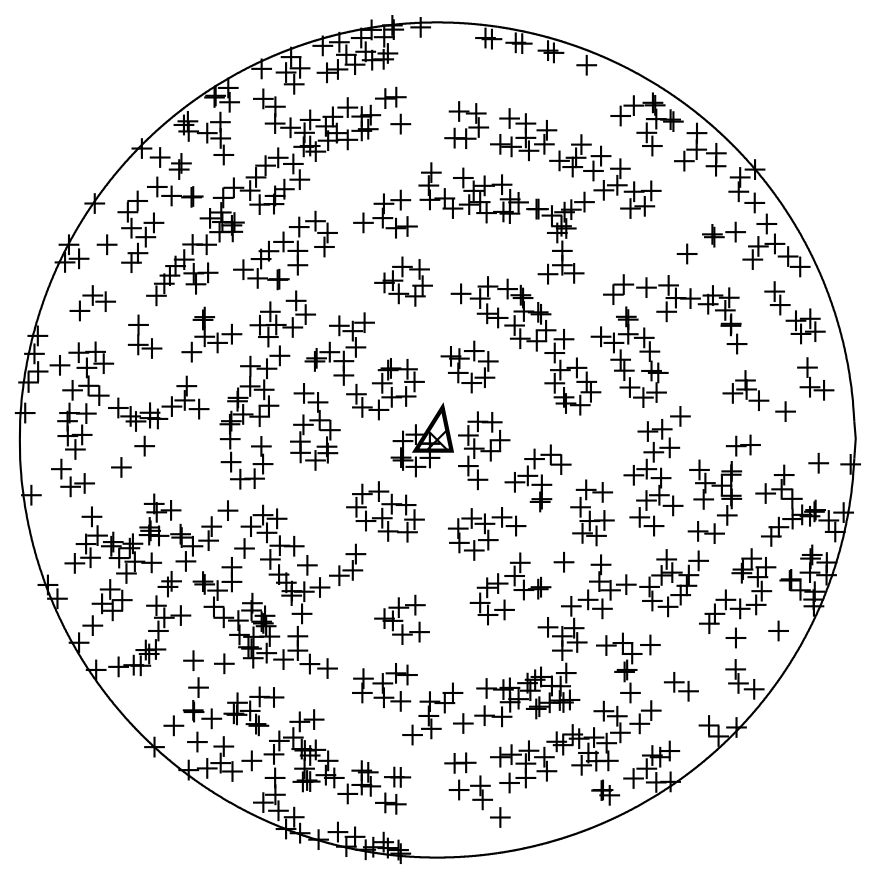

Figure 11: Triangular plate used for AISM and FEM simulations with the truncation parameter $\gamma=22.44$. $\ldots$, plate boundaries; + , sources; $\times$, receiver; $\_$, truncature.

Fig. 12 shows the modulus and the phase of $G_{\Omega}$ estimated from AISM and FEM, and the associated error (Eq. (48), where $G_{\Omega}^{(\text {ref })}$ is taken as the FEM estimation). The results exhibit a similar behaviour to those presented in Fig. 10. Large fluctuations in the modulus and on the phase lead to a considerable increase in the error, which is mainly due to the inability of the method to predict a strong resonant behaviour with a finite number of image sources. However, the accuracy of AISM is improved with frequency as the effects of structural damping are dominant over resonances of the system.

Furthermore, for estimating the vibrational behaviour of a plate, FEM needs the computation of the 


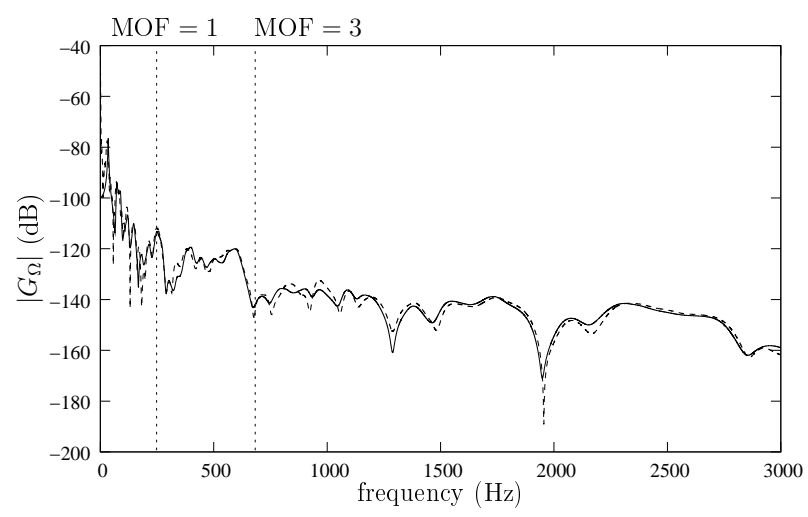

(a)

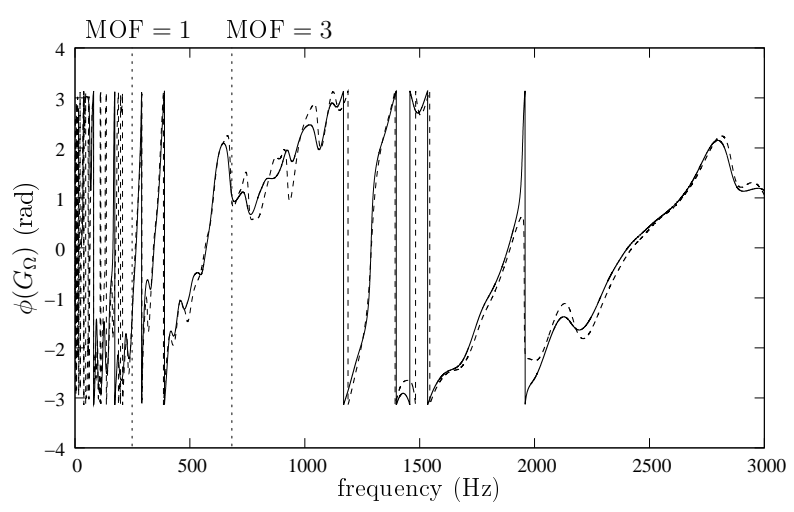

(b)

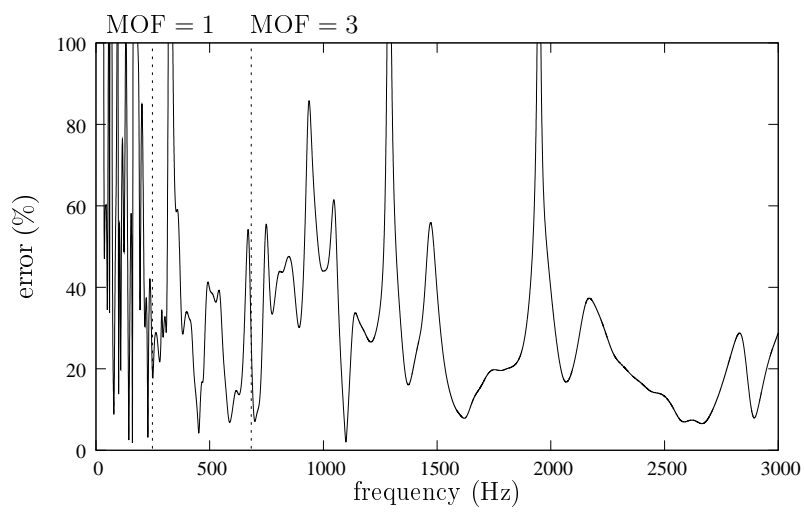

(c)

Figure 12: ISM and FEM simulations for the arbitrary polygonal plate of Fig. 11 for the damping ratio $\eta=0.07$. (a) modulus, (c) phase and (e) error. — , FEM solution; ----, AISM.

field on all the nodes of the model, while in AISM it is exclusively obtained at the observation point, allowing faster computations. As an example, for obtaining the responses shown in Fig. 12, AISM was approximately 40 times faster than FEM. The difference in terms of computation time is also linked to the simplicity of the AISM calculations, which consist in geometrical transformations for generating the image source cloud and then on a sum of the contributions of the sources for constructing the vibrational field at the receiver.

\subsection{Influence of the truncation radius on the accuracy of AISM}

The aim of this subsection is to study the convergence of AISM when increasing the truncation radius. For the purpose of predicting the mean value of the response of complex vibrating systems, Skudrzyk [24] pointed out that, for a plate with a driver or a receiver near an edge, the contribution of the nearest image source is usually strong enough to mask the reverberant field, and that including the effect of more image sources only sharpens the minima of the response curve. Fig. 13 shows the comparison between AISM estimation and exact Green's function $G_{\Omega}$ for the isosceles right triangular plate (Fig. 8) with $\eta=0.03$, for two truncation radii, given by $\gamma=6.15$ (52 sources) and $\gamma=30.74$ (1255 sources). 


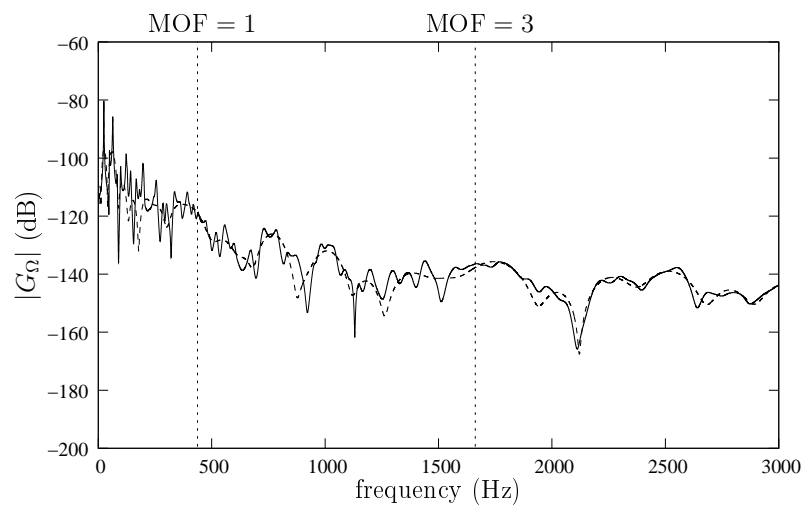

(a)

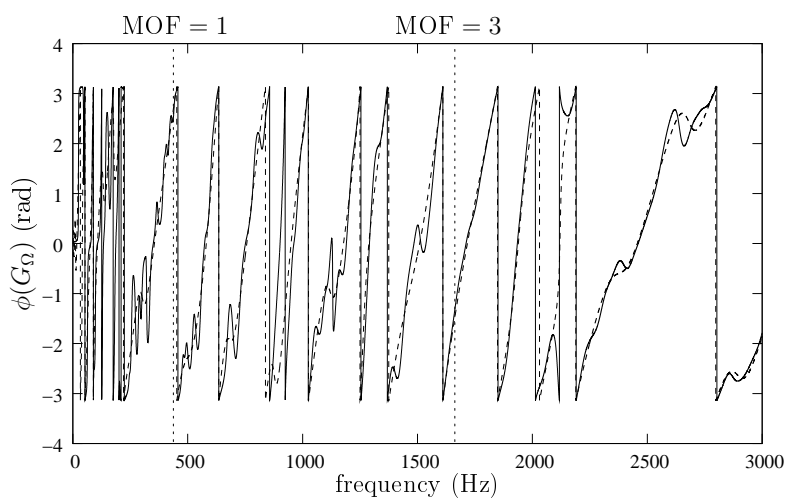

(c)

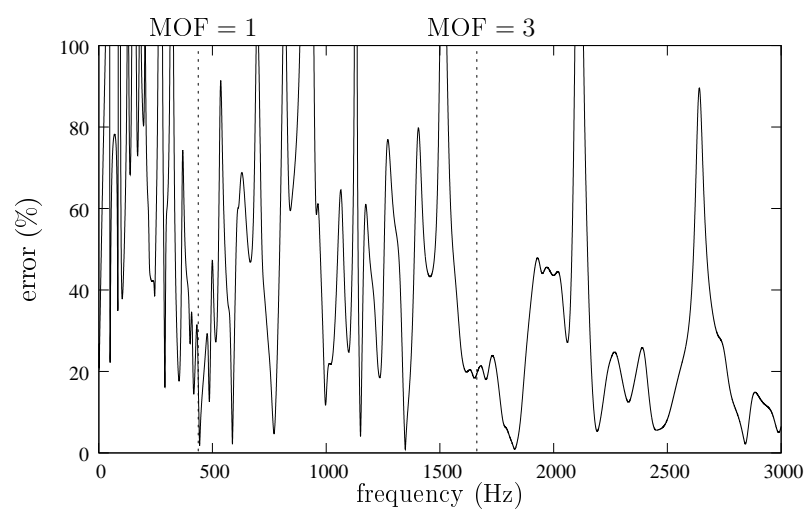

(e)

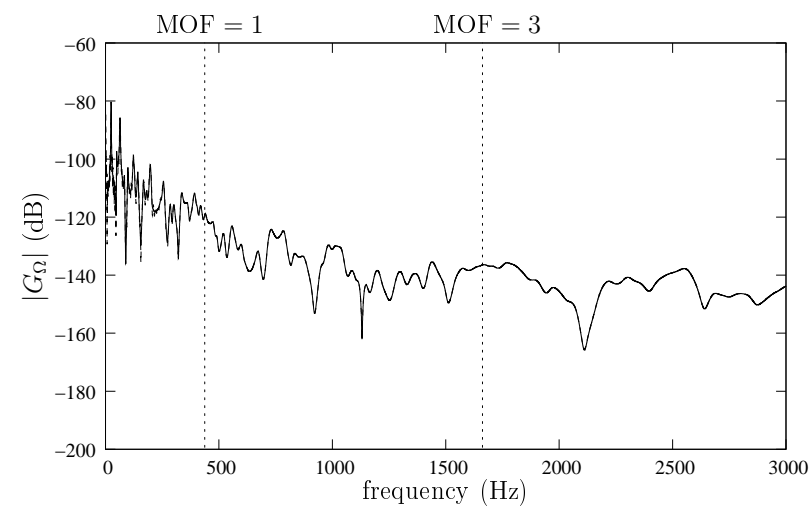

(b)

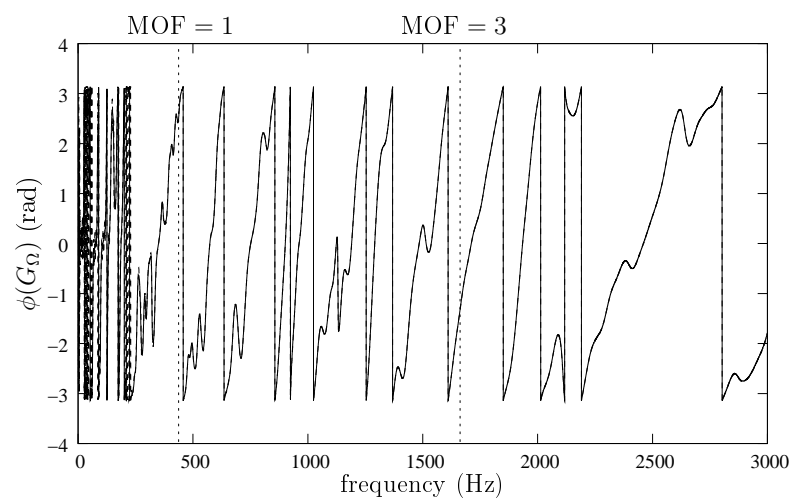

(d)

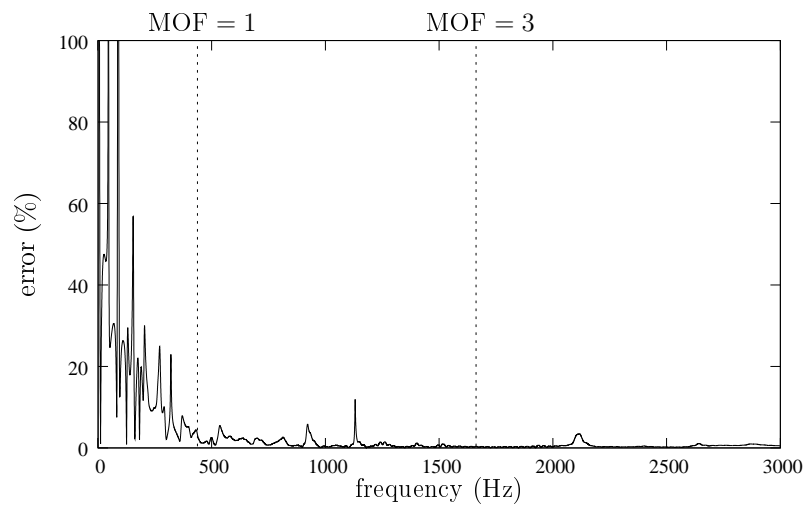

(f)

Figure 13: Frequency response of the isosceles triangular plate for two values of the truncation parameter: (a) modulus, (c) phase and (e) error for $\gamma=6.15$; (b) modulus, (d) phase and (f) error for $\gamma=30.74$. - , exact solution; ----, AISM. 
The value $\gamma=6.15$ leads to an estimation of a mean line of the response and, $\gamma=30.74$ leads to a low error in medium and high frequency ranges. Thus, the accuracy can be controlled by broadening the cloud of image sources. Fig. 14 shows the error on the estimation of $\left|G_{\Omega}\right|$ averaged over the high frequency band (ending at $3 \mathrm{kHz}$ ) as a function of $\gamma$, from which the limit $\gamma \rightarrow \infty$ leading to the exact solution is confirmed.

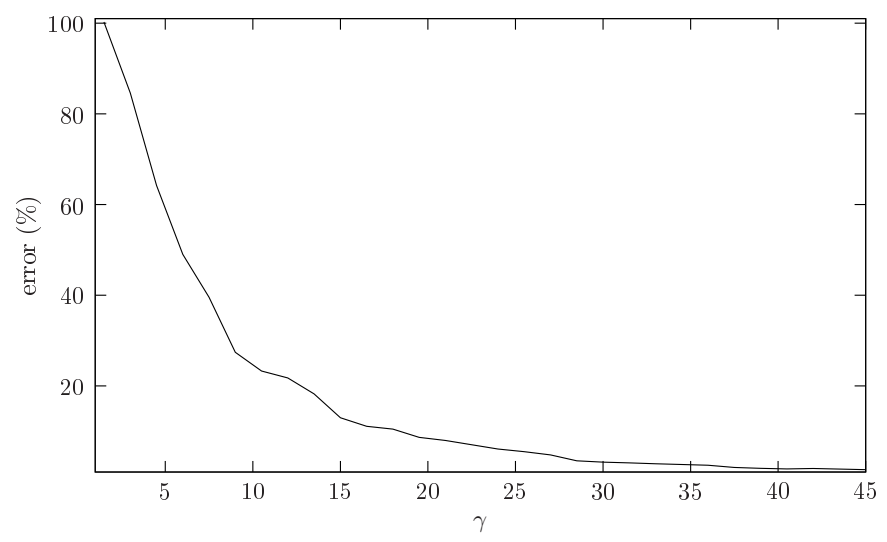

Figure 14: Error on the estimation of $\left|G_{\Omega}\right|$ as a function of $\gamma$ for the isosceles right triangular plate, averaged over the high-frequency band.

\section{Conclusion}

The Image Source Method (ISM) is a deterministic method that allows to calculate Green's functions for the flexural vibrations of simply supported convex polygonal plates. Based on this, we have exposed the general method for obtaining such Green's functions. Particular plate geometries leading to a spatially periodic image source cloud yield a spatially periodic Green's function, which can be expressed as a modal expansion by means of a Poisson summation formula. In this way, we have obtained new Green's functions for plates of particular geometries, i.e. rectangle, isosceles right triangle, half-equilateral triangle and equilateral triangle. Subsequently, we have developed a tool for predicting the flexural vibrations of arbitrarily shaped convex polygonal plates, called the Approximate Image Source Method (AISM), which consists in truncating the image source cloud. The results show a convergence towards the exact or the FEM solutions as the truncation radius is increased, since more image sources are taken into account. In classical modal methods, the number of modes to take into account needs to be increased for high frequencies and for a high damping ratio. On the contrary, since AISM is based on wave propagation, its accuracy is improved in high frequencies, where structural damping is predominant. In counterpart, AISM needs a large number of image sources for the analysis of highly reverberant fields since rapid amplitude and phase variations over frequency are present. However, with few sources in the truncation radius, AISM proves to be able to predict the mean line of the response. Finally, only simply supported boundaries have been considered, for which the reflection coefficient is unitary and omnidirectional. ISM can then be applied to plates with roller boundary conditions, but other kinds of boundaries need further investigation. Coupled structures, such as plate assemblies or stiffened plates [25] can also be investigated by adapting principles of ISM presented in this paper. 


\section{A Construction of the image source cloud}

For a given edge $E_{p}$ delimited by vertices $V_{p}$ and $V_{p+1}$, the location of the image source $S_{m, p}$ relatively to the location of its so-called mother source $S_{m}$ is determined by vector $\mathbf{S}_{m} \mathbf{S}_{m, p}$, which can be written as

$$
\mathbf{S}_{m} \mathbf{S}_{m, p}=2\left(\mathbf{S}_{m} \mathbf{V}_{p}+\mathbf{V}_{p} \mathbf{H}_{p}\right)
$$

where $H_{p}$ is the intersection point of vectors $\mathbf{S}_{m} \mathbf{S}_{m, p}$ and $\mathbf{V}_{p} \mathbf{V}_{p+1}$, as shown in Fig. 15 for the construction of source $S_{1}$ from source $S_{0}$ and edge $E_{1}$. Using absolute coordinates, the location of image source $S_{m, p}$ is described by vector $\mathbf{r}_{m, p}$, yielding

$$
\mathbf{r}_{m, p}=-\mathbf{r}_{m}+2 \mathbf{v}_{p}+2 \frac{\left(\mathbf{r}_{m}-\mathbf{v}_{p}\right) \cdot\left(\mathbf{v}_{p+1}-\mathbf{v}_{p}\right)}{\left|\mathbf{v}_{p+1}-\mathbf{v}_{p}\right|^{2}}\left(\mathbf{v}_{p+1}-\mathbf{v}_{p}\right)
$$

where $\mathbf{r}_{m}$ is the location of the mother source and $\mathbf{v}_{p}$ and $\mathbf{v}_{p+1}$ are the locations of the vertices defining $E_{p}$.

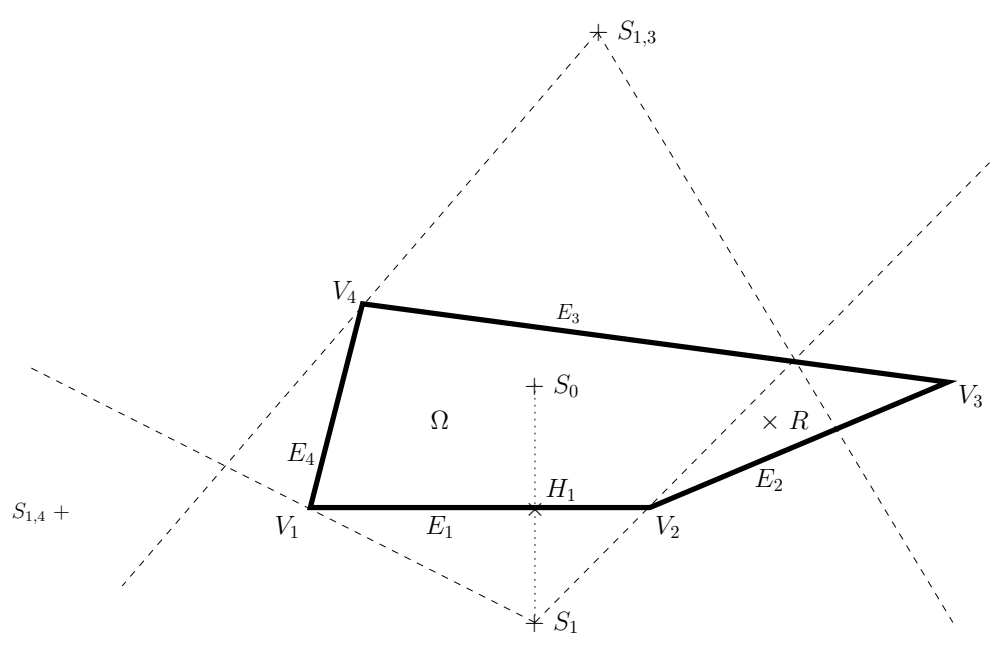

Figure 15: Example of image source geometrical construction. _- plate boundaries; +, sources; $\times$, receiver; ---, light zones limits.

The amplitude weights of the sources depend on the reflection coefficient of the boundaries and on the number $n$ of reflections that lead to the source. Thus, for a plate with reflection coefficient $\mathcal{R}$, its amplitude weight is

$$
A=(\mathcal{R})^{n} .
$$

The reflection coefficient of simply supported edges is $\mathcal{R}=-1$, yielding

$$
A=(-1)^{n} \text {. }
$$

For example, in Fig. 15, the amplitude weigths of sources $S_{0}, S_{1}$ and $S_{1,3}$ are respectively $A_{0}=1$, $A_{1}=-1$ and $A_{1,3}=1$.

Furthermore, a given source contributes to the field at the receiver if it is visible from the receiver, i.e. if the receiver is in the "light" zone of the source. Additionally, a given source creates a new image source if the inside (i.e. the reflecting side) of the corresponding edge is in its light zone. The same can be interpreted in terms of rays. Each image source contribution describes a vibrational ray in the plate. Thus, the existence of an image source relies on whether the corresponding ray path between the source and the receiver is possible or not. However, some precautions are to be taken into account since an image source that is invisible from the receiver can give rise to a visible image source. Fig. 15 shows an example of construction of the first image sources of a plate, in which some image sources do not contribute to the vibrational field at the receiver. $S_{1,2}$ does not exist because edge $E_{2}$ is out of the light zone of $S_{1}$. Moreover, $S_{1}$ does not contribute to the vibrational field at $R$, but its image source at $E_{3}, S_{1,3}$, does. 


\section{B Elementary cells of equilateral and half-equilateral triangular plates}

\section{B.1 Half-equilateral triangular plate}

Fig. 16 shows the elementary cell of the half equilateral triangular plate.

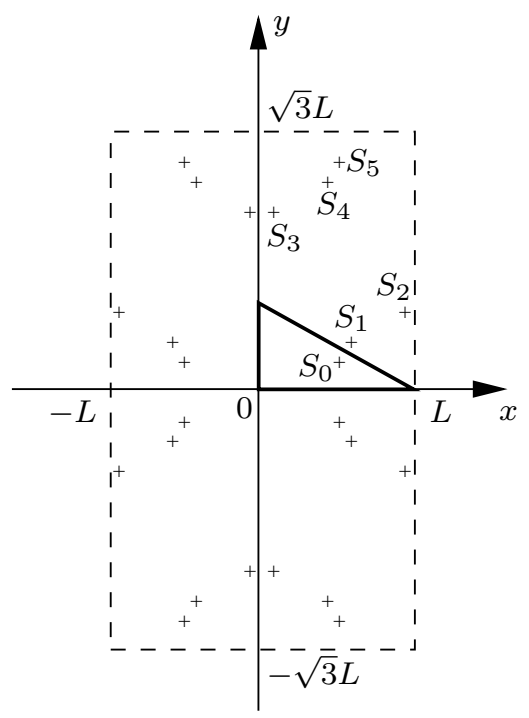

Figure 16: Elementary cell of the half-equilateral triangular plate. Six sources, denoted $S_{i}(i=0, \ldots, 5)$ and located at $\left(x_{i}, y_{i}\right)$, are necessary to describe this cell.

The coordinates of the principal source of each of the six quadrupoles that form this elementary cell (presented in section 2.5.3) are

$$
\begin{aligned}
& \left(\begin{array}{l}
x_{0} \\
y_{0}
\end{array}\right) \quad \text { original source } \\
& \left(\begin{array}{l}
x_{1} \\
y_{1}
\end{array}\right)=\frac{1}{2}\left(\begin{array}{c}
L+x_{0}-\sqrt{3} y_{0} \\
-y_{0}+\sqrt{3}\left(L-x_{0}\right)
\end{array}\right) \\
& \left(\begin{array}{l}
x_{2} \\
y_{2}
\end{array}\right)=\frac{1}{2}\left(\begin{array}{c}
L+x_{0}+\sqrt{3} y_{0} \\
y_{0}+\sqrt{3}\left(L-x_{0}\right)
\end{array}\right) \\
& \left(\begin{array}{l}
x_{3} \\
y_{3}
\end{array}\right)=\frac{1}{2}\left(\begin{array}{c}
L-x_{0}-\sqrt{3} y_{0} \\
-y_{0}+\sqrt{3}\left(L+x_{0}\right.
\end{array}\right) \\
& \left(\begin{array}{l}
x_{4} \\
y_{4}
\end{array}\right)=\frac{1}{2}\left(\begin{array}{c}
L-x_{0}+\sqrt{3} y_{0} \\
y_{0}+\sqrt{3}\left(L+x_{0}\right)
\end{array}\right) \\
& \left(\begin{array}{l}
x_{5} \\
y_{5}
\end{array}\right)=\left(\begin{array}{c}
L-x_{0} \\
\sqrt{3} L-y_{0}
\end{array}\right),
\end{aligned}
$$

where $L$ is the length of the triangle base. The sequence giving the relative weights of the quadrupoles that form the elementary cell is, in the same order,

$$
\xi_{i}=\{1,-1,1,1,-1,1\} .
$$

\section{B.2 Equilateral triangular plate}

Fig. 17 shows the elementary cell of the equilateral triangular plate. 


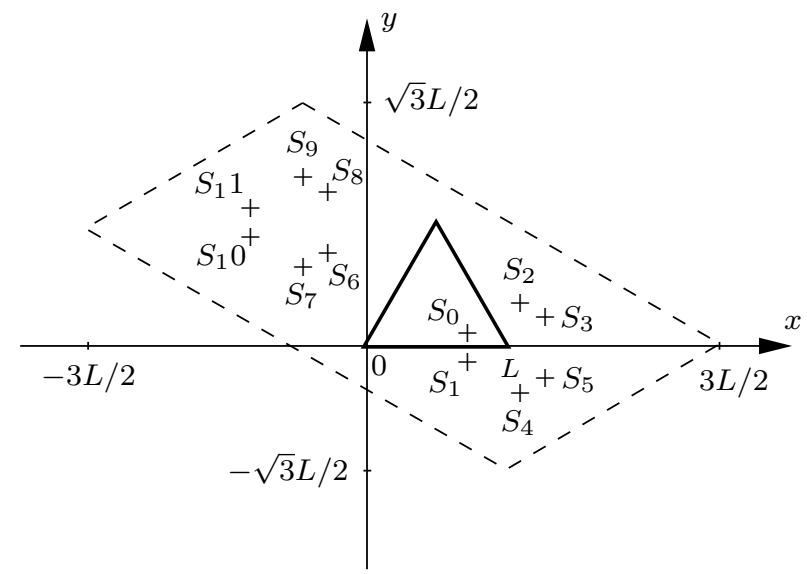

Figure 17: Elementary cell of the equilateral triangular plate. Twelve sources, denoted $S_{i}(i=0, \ldots, 11)$ and located at $\left(x_{i}, y_{i}\right)$, are necessary to describe this cell.

The coordinates of the sources of the the elementary cell (section 2.5.4) of this plate are

$$
\begin{aligned}
\left(\begin{array}{l}
x_{0} \\
y_{0}
\end{array}\right) & \text { original source } \\
\left(\begin{array}{l}
x_{1} \\
y_{1}
\end{array}\right) & =\left(\begin{array}{c}
x_{0} \\
-y_{0}
\end{array}\right) \\
\left(\begin{array}{l}
x_{2} \\
y_{2}
\end{array}\right) & =\frac{1}{2}\left(\begin{array}{c}
3 L-\sqrt{3} y_{0}-x_{0} \\
\sqrt{3} L+y_{0}-\sqrt{3} x_{0}
\end{array}\right) \\
\left(\begin{array}{l}
x_{3} \\
y_{3}
\end{array}\right) & =\frac{1}{2}\left(\begin{array}{c}
3 L+\sqrt{3} y_{0}-x_{0} \\
\sqrt{3} L-y_{0}-\sqrt{3} x_{0}
\end{array}\right) \\
\left(\begin{array}{l}
x_{4} \\
y_{4}
\end{array}\right) & =\frac{1}{2}\left(\begin{array}{c}
3 L-\sqrt{3} y_{0}-x_{0} \\
-\sqrt{3} L-y_{0}+\sqrt{3} x_{0}
\end{array}\right) \\
\left(\begin{array}{l}
x_{5} \\
y_{5}
\end{array}\right) & =\frac{1}{2}\left(\begin{array}{c}
3 L+\sqrt{3} y_{0}-x_{0} \\
-\sqrt{3} L+y_{0}+\sqrt{3} x_{0}
\end{array}\right) \\
\left(\begin{array}{l}
x_{6} \\
y_{6}
\end{array}\right) & =\frac{1}{2}\left(\begin{array}{c}
\sqrt{3} y_{0}-x_{0} \\
y_{0}+\sqrt{3} x_{0}
\end{array}\right) \\
\left(\begin{array}{l}
x_{7} \\
y_{7}
\end{array}\right) & =\frac{1}{2}\left(\begin{array}{c}
-\sqrt{3} y_{0}-x_{0} \\
-y_{0}+\sqrt{3} x_{0}
\end{array}\right) \\
\left(\begin{array}{l}
x_{8} \\
y_{8}
\end{array}\right) & =\frac{1}{2}\left(\begin{array}{c}
\sqrt{3} y_{0}-x_{0} \\
2 \sqrt{3} L-y_{0}-\sqrt{3} x_{0}
\end{array}\right) \\
\left(\begin{array}{l}
x_{9} \\
y_{9}
\end{array}\right) & =\frac{1}{2}\left(\begin{array}{c}
-\sqrt{3} y_{0}-x_{0} \\
2 \sqrt{3} L+y_{0}-\sqrt{3} x_{0}
\end{array}\right) \\
\left(\begin{array}{l}
x_{10} \\
y_{10}
\end{array}\right) & =\frac{1}{2}\left(\begin{array}{l}
-3 L+2 x_{0} \\
\sqrt{3} L-2 y_{0}
\end{array}\right) \\
\left(\begin{array}{l}
x_{11} \\
y_{11}
\end{array}\right) & =\frac{1}{2}\left(\begin{array}{l}
-3 L+2 x_{0} \\
\sqrt{3} L+2 y_{0}
\end{array}\right), \\
& =1
\end{aligned}
$$

where $L$ is the length of the triangle side. The sequence giving the relative weights of the sources in the 
elementary cell is, in the same order,

$$
\xi_{i}=\{1,-1,-1,1,1,-1,-1,1,1,-1,-1,1\} .
$$

\section{References}

[1] R.H. Lyon, R.G. Dejong, Theory and Application of Statistical Energy Analysis, ButterworthHeinemann, Boston, 1995.

[2] B.R. Mace, D. Duhamel, M.J. Brennan, L. Hinke, Finite element prediction of wave motion in structural waveguides, Journal of the Acoustical Society of America 117(5) (2005) 2835-2843.

[3] A. Le Bot, A vibroacoustic model for high frequency analysis, Journal of Sound and Vibration 211(4) (1998) 537-554.

[4] V. Cotoni, P. Shorter, R. Langley, Numerical and experimental validation of a hybrid finite elementstatistical energy analysis method, Journal of the Acoustical Society of America 122(1) (2007) 259270.

[5] K.-S. Chae, J.-G. Ih, Prediction of vibrational energy distribution in the thin plate at high-frequency bands by using the ray tracing method, Journal of Sound and Vibration 240(2) (2001) 263-292.

[6] P. Ladevèze, The variational theory of complex rays for the calculation of medium-frequency vibrations, Engineering with Computers 18 (1999) 193-214.

[7] P. Rouch, P. Ladevèze, The variational theory of complex rays: a predictive tool for medium frequency vibrations, Computer Methods in Applied Mechanics and Engineering, 192 (2003) 3301-3315.

[8] J.B. Allen, D.A. Berkley, Image method for efficiently simulating small-room acoustics, Journal of the Acoustical Society of America 65(4) (1979) 943-950.

[9] J. Borish, Extension of the image model to arbitrary polyhedra, Journal of the Acoustical Society of America 75(6) (1984) 1827-1836.

[10] L. Cremer, H.A. Müller, Principles and applications of room acoustics, Applied Science, London, 1982.

[11] H. Kuttruff, Room acoustics, Applied Science, London, 1973.

[12] F.P. Mechel, Improved mirror source method in room acoustics, Journal of Sound and Vibration 256(6) (2002) 873-940.

[13] K.F. Graff, Wave motion in elastic solids, Oxford University Press, 1975.

[14] D.J. Gorman, Vibration Analysis of Plates by the Superposition Method, World Scientific, Singapore, 1999.

[15] J. Brunskog, Image solution for clamped finite beams, Journal of Sound and Vibration 287(4-5) (2005) 1057-1064.

[16] R. Gunda, S.M. Vijayakar, R. Singh, Method of images for the harmonic response of beams and rectangular plates, Journal of Sound and Vibration 185(2) (1995) 791-808.

[17] Z.Y. Huang, W.K. Jiang, An efective method calculating acoustic Green's function for closed rectangular cavity using the Ewald's summation technique, Acta Acustica united with Acustica 93 (2007) 853-856. 
[18] A. Hayir, I. Bakirtas, A note on a plate having a circular cavity excited by plane harmonic SH waves, Journal of Sound and Vibration 271 (2004) 241-255.

[19] C.Hu, X.-Q. Fang, W.-H. Huang, Multiple scattering of flexural waves in a semi-infinite thin plate with a cutout, International Journal of Solids and Structures 44 (2007) 436-446.

[20] P.M. Morse, H. Feshbach, Methods of theoretical physics, McGraw-Hill, New York, 1953.

[21] R. Szilard, Theories and Applications of Plate Analysis: Classical, Numerical and Engineering Methods, John Wiley and sons, New York, 2004.

[22] G. Rabbiolo, R.J. Bernhard, F.A. Milner, Definition of a high-frequency threshold for plates and acoustical spaces, Journal of Sound and Vibration 277 (2004) 647-667.

[23] M.R. Schroeder, Statistical parameters of the frequency response curves of large rooms, Journal of the Audio Engineering Society 35 (1987) 299-306.

[24] E. Skudrzyk, The mean-value method of predicting the dynamic response of complex vibrators, Journal of the Acoustical Society of America 67(4) (1980) 1105-1135.

[25] J.R.F. Arruda, F. Gautier, L.V. Donadon, Computing reflection and transmission coefficients for plate reinforcement beams, Journal of Sound and Vibration 307 (2007) 564-577. 